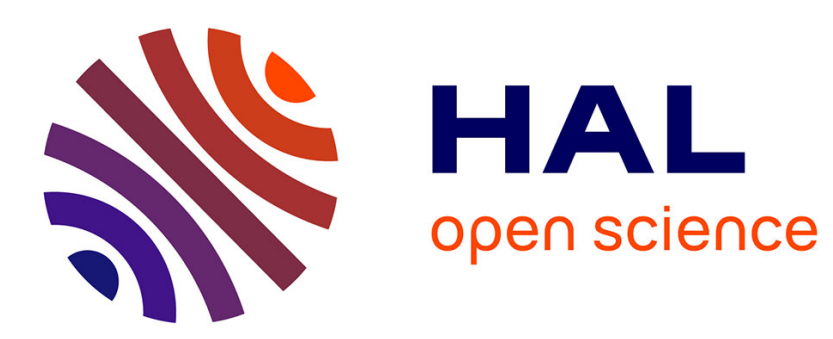

\title{
Les céramiques de l'oppidum laténien de la Pierre d'Appel à Étival-Clairefontaine (Vosges)
}

\author{
Marc Dalaut, Alain Deyber
}

\section{To cite this version:}

Marc Dalaut, Alain Deyber. Les céramiques de l'oppidum laténien de la Pierre d'Appel à ÉtivalClairefontaine (Vosges). Gallia - Fouilles et monuments archéologiques en France métropolitaine, 1988, 45, pp.29-47. 10.3406/galia.1987.2878 . hal-01934398

\section{HAL Id: hal-01934398 \\ https://hal.science/hal-01934398}

Submitted on 14 Jan 2020

HAL is a multi-disciplinary open access archive for the deposit and dissemination of scientific research documents, whether they are published or not. The documents may come from teaching and research institutions in France or abroad, or from public or private research centers.
L'archive ouverte pluridisciplinaire HAL, est destinée au dépôt et à la diffusion de documents scientifiques de niveau recherche, publiés ou non, émanant des établissements d'enseignement et de recherche français ou étrangers, des laboratoires publics ou privés.

\section{(1) (1) $\$$}

Distributed under a Creative Commons Attribution - NonCommercial - NoDerivatives $\mid 4.0$ 


\title{
LES CÉRAMIQUES DE L'OPPIDUM LATÉNIEN DE LA PIERRE D'APPEL A ÉTIVAL-CLAIREFONTAINE (VOSGES)
}

\author{
Par Marc DALAUT et Alain DEYBER
}

L'oppidum de La Pierre d'Appel d'ÉtivalClairefontaine (Vosges) domine un carrefour des principales voies transvosgiennes et l'un des principaux points de passage obligés sur la HauteMeurthe. Les fouilles archéologiques conduites de 1967 à 1981 ont révélé trois établissements laténiens s'échelonnant de la fin de La Tène C à La Tène D2 dans la chronologie de Reinecke et de Drack ${ }^{1}$.

L'intérêt du site réside dans la stratification des structures et des vestiges sur une épaisseur atteignant par endroits plusieurs mètres. Même si une partie de l'habitat a seulement été dégagée, les recherches ont permis de rassembler un important matériel en situation de fouille. Pour cette raison, il devenait indispensable d'exploiter et de publier ce qui pouvait l'être. Cette étude résulte de l'exploitation du "tessonier" et des treize rapports de fouilles rédigés de 1967 à 1981 . Il n'en reste pas moins qu'elle doit être considérée - pour employer le vocabulaire de la statistique - comme un sondage, et que les explications proposées ne sont pas des certitudes péremptoires mais des recherches de solutions aux problèmes rencontrés: bref, des hypothèses de travail, susceptibles d'être modifiées par toute importante découverte ultérieure ou le progrès des techniques de fouille et des méthodes d'analyse.

1 La stratigraphie du site compte trois niveaux, le niveau I étant le plus récent. Archéologiquement ces niveaux sont subdivisés mais une chronologie aussi fine n'est pas utilisable pour l'étude du matériel céramique qui sera, ici, simplement réparti selon les niveaux I, II et III.
La céramique est l'un des principaux matériaux archéologiques de La Pierre d'Appel. Mais cette étude porte uniquement sur la poterie, c'est-à-dire la vaisselle de terre cuite, fabriquée localement ou importée dans le cadre d'un commerce pratiqué parfois à longue distance. La question des autres vestiges en céramique n'est pas abordée ici (crécelles, jetons, plaques de foyer, etc.). Les multiples données rassemblées ont permis une description statistique élémentaire, portant principalement sur les techniques de fabrication, les formes ( 7 groupes) et la décoration ( 6 types). Nous nous sommes attachés le plus souvent possible aux détails concrets : aussi, les réflexions sont-elles aussi bien dictées par les préoccupations du potier que par celles de l'archéologue ou de l'historien ${ }^{2}$.

Enfin, l'oppidum de La Pierre d'Appel est replacé dans un cadre plus vaste : le Bassin rhénan, les sites allemands et tchécoslovaques et ceux du Centre et du Midi de la France (fig. 1 et annexe). L'étude de la diffusion des formes céramiques de $\mathrm{La}$ Pierre d'Appel permet de situer cette production dans le contexte plus large des civilisations de La Tène moyenne et finale.

2 Voir le détail des recherches (calculs statistiques, bibliographie ...) dans M. Dalaut, La céramique laténienne de la Pierre d'Appel, Etival-Clairefontaine (Vosges), Mémoire de Maitrise, Faculté des Lettres et Sciences Humaines, Nancy II, $1982, n^{\circ} \mathrm{N} 82$, et la synthèse de l'ensemble des découvertes effectuées sur le site dans A. DEYBER, avec la collaboration de M. Dalaut, E. Ladier, A. Weisrock, L'habitat fortifiè laténien de La Pierre d'Appel à Étival-Clairefontaine (Vosges), Gallia, 42, 1984, p. 175-217. 


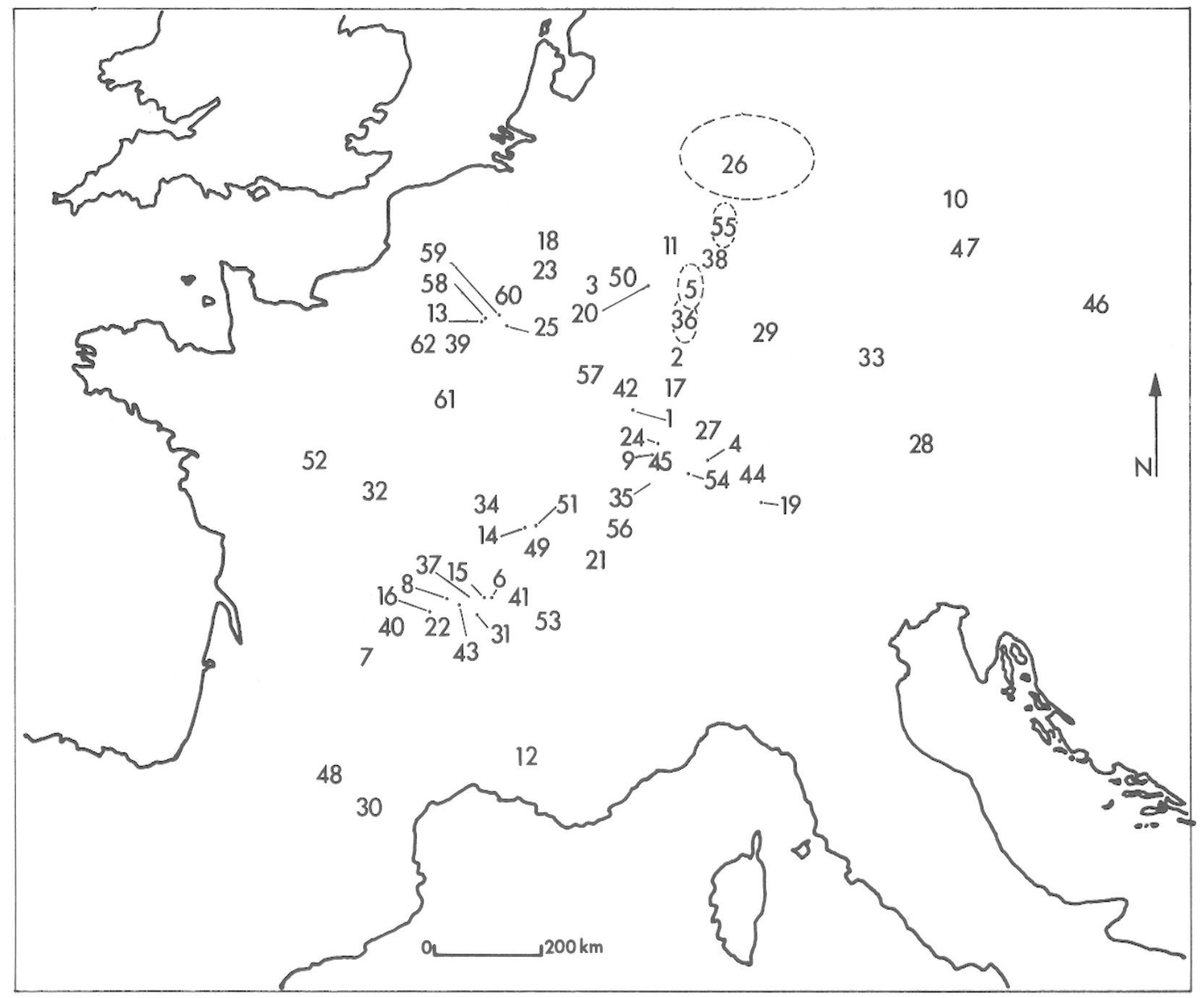

Fig. 1 - Situation de La Pierre d'Appel et des sites de référence.

1: La Pierre d'Appel (Vosges);

2: Achenheim-Oberschaeffolsheim (Bas-Rhin);

3 : Die Altburg von Bundenbach (Allemagne, Rheinl. Pfalz);

4 : Altenburg (Allemagne, $\mathrm{Kr}$. Waldshut);

5 : Alzey et ses environs (Allemagne, Rheinl. Pfalz);

6 : Amplepuis, Le Terrail (Rhône);

7 : Argentat, Le Puy-du-Tour (Corrèze);

8: Aulnat (Puy-de-Dôme);

9 : Bâle (Suisse);

10 : Bilina et Radovesice (Tchécoslovaquie, Bohême);

11 : Braubach (Allemagne, Rheinl. Pfalz);

12: Cavaillon (Vaucluse);

13 : Celles-les-Condé (Aisne);

14 : Chalon-sur-Saône (Saône-et-Loire);

15 : Chambles, Essalois (Loire);

16 : Clermont-Ferrand (Puy-de-Dôme);

17 : Ehl (Bas-Rhin);

18: Éprave, Le Trou de l'Ambre (Belgique, province de Namur);

19 : Eschen (Liechtenstein);

20 : Frohnhausen (Allemagne, Kr. Birkenfeld);

21: Genève (Suisse);

22 : Gergovie (Puy-de-Dôme);

23 : Göblingen-Nospelt (Luxembourg);

24 : Habsheim (Bas-Rhin);

25 : Hauviné (Ardennes);

26 : Hesse supérieure, région de Starkenburg (Allemagne);

27 : Hüfingen (Allemagne, Bad. Württ., Kr. Donaueschingen);

28: Karlstein (Allemagne, Bavière);

29 : Ingelfingen (Allemagne, Bad. Württ. Kr. Kunselsau.);

30: La Lagaste (Aude);

31 : Lijay (Loire);
32: Levroux (Indre);

33 : Manching (Allemagne, Ldkr. Ingolstadt);

34 : Mont-Beuvray (Saône-et-Loire);

35 : Mont-Terrible (Suisse);

36 : Palatinat (Allemagne);

37 : Roanne (Loire);

38 : Russelsheim-am-Main (Allemagne);

39 : Sablonnière (Seine-et-Marne);

40 : Saint-Fréjoux (Corrèze);

41 : Saint-Marcel-de-Felines, Le Crêt Chatelard (Loire);

42 : Saint-Maurice-aux-Forges (Meurthe-et-Moselle);

43 : Saint-Maurice-sur-Loire, Jœuvre (Loire);

44 : Schänis (Suisse);

45 : Sissach-Brühl (Suisse);

46 : Stare-Hradisko (Tchécoslovaquie, Bohême);

47 : Stradonice (Tchécoslovaquie, Bohême);

48 : Toulouse (Haute-Garonne);

49 : Tournus (Saône-et-Loire);

50 : Trèves (Allemagne, Rheinl. Pfalz);

51 : Verdun-sur-le-Doubs, Le Petit Chauvort (Saône-et-Loire);

52 : Vernou-sur-Brenne (Indre-et-Loire);

53 : Vienne (Isère);

54 : Vindonissa (Suisse);

55 : Le Wetterau (Allemagne, Hesse);

56 : Yverdon (Suisse);

57 : Sion (Meurthe-et-Moselle);

58 : Villeneuve-Saint-Germain (Aisne);

59: Condé-sur-Suippe-Variscourt (Aisne);

60 : Menil-Annelles et Ville-sur-Retourne (Ardennes);

61 : Pont-sur-Yonne (Yonne);

62 : Vaires-sur-Marne (Seine-et-Marne); 


\section{LES TECHNIQUES DE FABRICATION}

\section{LES ÉTAPES DE LA FABRICATION}

La céramique indigène de La Pierre d'Appel présente un éventail limité de caractéristiques techniques.

Des analyses en laboratoire ont démontré que la pâte céramique était généralement constituée d'une argile très pure provenant de gisements naturels locaux. Les poteries fines (poterie peinte et "grise lissée») sont façonnées à partir d'une argile très peu ferrugineuse qui devient presque blanche après cuisson en atmosphère oxydante, alors que dans les mêmes conditions, la pâte des céramiques communes offre une couleur rougeâtre.

La présence de dégraissants minéraux dans la plupart des poteries est indiscutable : il s'agit de petits grains de quartz ou de mica. Si les éléments les plus fins faisaient sans doute déjà partie intégrante de l'argile, les plus importants ont été probablement ajoutés. La proportion de dégraissant mélangé à l'argile est moyenne : de 15 à $30 \%$, la dimension des grains varie, des plus fins $(0,05 \mathrm{~mm})$ aux plus grossiers (plus de $0,5 \mathrm{~mm}$ ), selon le soin pris pour tamiser le dégraissant. Les dégraissants les plus grossiers se trouvent dans les poteries communes : la tranche des tessons présente souvent un aspect grumeleux et quelque peu pulvérulent. La présence de dégraissants végétaux dans la pâte de certaines poteries est plausible mais ne peut être confirmée en l'absence d'analyses scientifiques.

Pour le façonnage, deux techniques ont été employées : le colombin et le tour. Pour les vases montés à la main, la méthode du colombin en spirale a été utilisée : chaque colombin est soudé au bout du précédent, comme si le vase devait être fabriqué à partir d'un seul boudin de terre. Les parois sont moins symétriques mais le vase est plus solide : l'aspect fonctionnel prime sur l'aspect esthétique. Quant au tournage, nous pouvons seulement observer que certains des tours utilisés devaient être bien équilibrés, et pouvoir atteindre une vitesse assez élevée, notamment ceux qui ont permis de façonner les poteries fines. Toutefois, le mode de façonnage n'est pas toujours discernable : le vase peut avoir été monté au colombin de façon très régulière ou été façonné sur un tour médiocre.

Au cours du séchage, opération fort délicate, le vase subissait les traitements de finition : raclage et tournassage, lissage, trempage dans un bain d'engobe, ou impression de décors estampés ou incisés. Si ce dernier type de finition ne répond qu'à un mobile esthétique, lissage et engobage correspondent à une nécessité fonctionnelle car ils permettent de pallier partiellement la porosité de la terre cuite.

Les modes de cuisson utilisés sont très variés et difficiles à déterminer. Les analyses scientifiques ont montré un large éventail des températures de cuisson. Pour la poterie fine, les températures oscillent de $750^{\circ} \mathrm{C}$ à $800^{\circ} \mathrm{C}$, ce qui suppose l'emploi de fours perfectionnés du type four à alandier et à sole perforée. Mais pour la poterie commune, les températures varient de $550^{\circ} \mathrm{C}$ (ce qui est tout juste suffisant) à $750^{\circ} \mathrm{C}$ : les températures les plus basses correspondent à des modes de cuisson primitifs, en plein air ou dans des fours rudimentaires. L'atmosphère de cuisson est très souvent oxydo-réductrice et il semble bien que la plupart des cuissons aient été menées sans contrôle délibéré de l'atmosphère, le seul but étant de parvenir aux températures nécessaires. Cependant, pour certaines catégories de vases, l'atmosphère était contrôlée, sans doute en phase terminale de cuisson : ainsi pour les poteries peintes et "grises lissées", l'atmosphère ne se révèle que très légèrement réductrice.

\section{ORIGINE DES PRODUCTIONS}

La variété des caractéristiques techniques traduit la diversité d'origine des productions. S'il est certain que les poteries fines proviennent de centres de fabrication importants et bien équipés, il est moins aisé de déterminer les parts de l'importation et de la production locale de la poterie commune.

Bien que les matières premières ne manquent pas, les conditions climatiques semblent exclure l'existence d'un centre de production important sur le site même. Ceci est confirmé, pour l'instant, par les fouilles qui ont en revanche révélé une aire de cuisson en plein air. Il est donc probable qu'il existait une production locale, peu développée, sans doute due à des artisans occasionnels, dans le cadre d'une économie domestique : de là proviennent sans doute les vases fabriqués selon les techniques les plus élémentaires. Ainsi, la plus grosse part des poteries communes a pu, elle aussi, être importée de centres de productions spécialisés comme celui de Bourgheim (Bas-Rhin) ${ }^{3}$.

3 F. PÉtry, Informations, Gallia, 38, 1980, p. 447-451. 


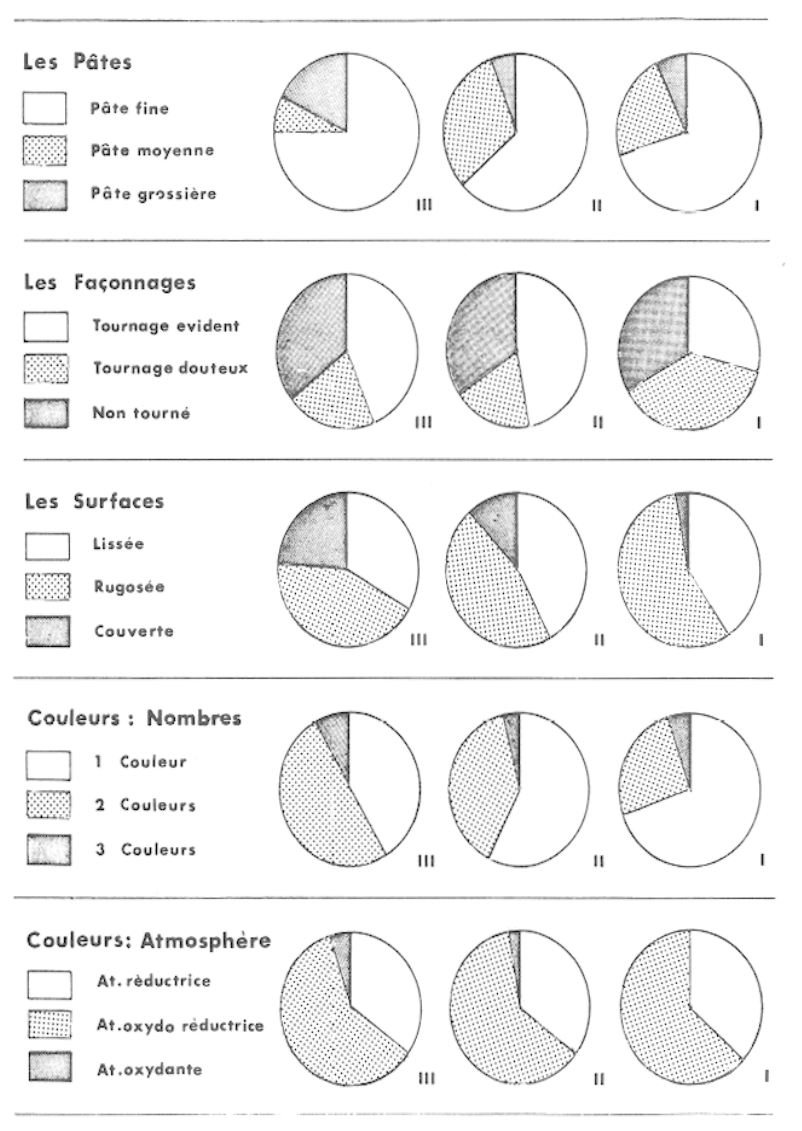

Fig. 2 - Techniques de fabrication et répartition selon les niveaux III à $\mathrm{I}$.

\section{Évolution}

Un sondage statistique portant sur 400 tessons, tous types de vases confondus donne, pour les trois périodes considérées, certaines indications quantitatives et évolutives (fig. 2). Si les techniques de fabrication sont soumises à des limitations technologiques, il semble que les potiers indigènes ont souvent su exploiter leurs connaissances et utiliser au mieux ces techniques : les pâtes sont majoritairement de bonne qualité, l'usage du tour est très fréquent ... La tendance, sur les trois niveaux, est à l'amélioration technique, faible dans les domaines de la préparation des pâtes et du façonnage, plus sensible pour la cuisson. La céramique fine est minoritaire mais se développe au cours des trois périodes. Cependant, au niveau I (le plus récent) tout se passe comme si la céramique de La Pierre d'Appel subissait une régression partielle et locale : le degré technologique est encore amélioré mais il semble que l'on utilise les perfectionnements techniques dans une moindre mesure, soit que l'on ait accordé moins de soins à la fabrication, soit que cette fabrication ait été le fait d'artisans moins qualifiés.
Pour expliquer ce phénomène nous pouvons avancer l'hypothèse suivante: aux niveaux III et II, la situation est comparable, la communauté d'habitants est largement ouverte aux influences extérieures, ce qui permet l'adoption de techniques étrangères, plus perfectionnées, et l'importation de céramique produite par des centres spécialisés. En revanche, au niveau I, il se produit un certain repli (économique, démographique) et l'oppidum vit beaucoup plus sur ses propres fabrications, toujours influencées par l'extérieur mais de moindre qualité car produites par des artisans moins expérimentés, sans doute non spécialisés.

\section{LES FORMES : GENNÉRALITÉS}

\section{Classification}

L'étude des formes (comme celle des décors) porte sur 605 vases d'origine indigène, toujours fragmentaires, reconstitués partiellement ou totalement. Une chronologie relative a pu être établie grâce à une stratigraphie assez fine.

Le mobilier céramique de La Pierre d'Appel présente une certaine homogénéité dans les formes, simples et purement fonctionnelles : aucune fantaisie, toutes ces formes sont à section circulaire horizontale, d'une silhouette très sobre (sauf exception, il n'existe pas d'éléments additionnels de préhension), de petite taille (hormis certains vases de stockage, aucun ne dépasse une trentaine de centimètres de hauteur).

La céramique de La Pierre d'Appel se limite à une poterie utilitaire destinée à trois fonctions : conservation et stockage des aliments, leur préparation et le couvert. Aucun ustensile particulier tel que mortier, poêlon ou lampe n'existe. Ce mobilier céramique est classé en sept groupes, définis d'aprè: l'emploi des récipients, leur technique de fabricatior et leur forme générale. Le classement en types qu. subdivise les catégories rend compte de certaines différences morphologiques. Ce classement a été établi par comparaison avec le mobilier céramique d'autres sites. La répartition en sept catégories, jattes (A), coupes (B), bouteilles $(\mathrm{C})$, marmites $(\mathrm{D})$, pots $(\mathrm{E})$, tonneaux $(\mathrm{F})$, dolia $(\mathrm{G})$, reprend celle des archéologues suisses et allemands.

\section{DEscription Catégorielle}

Catégorie $A$ : jattes (A1), écuelles et assiettes (A2), vases à boire (A3) (fig. 3 à 5 ) 
(a)
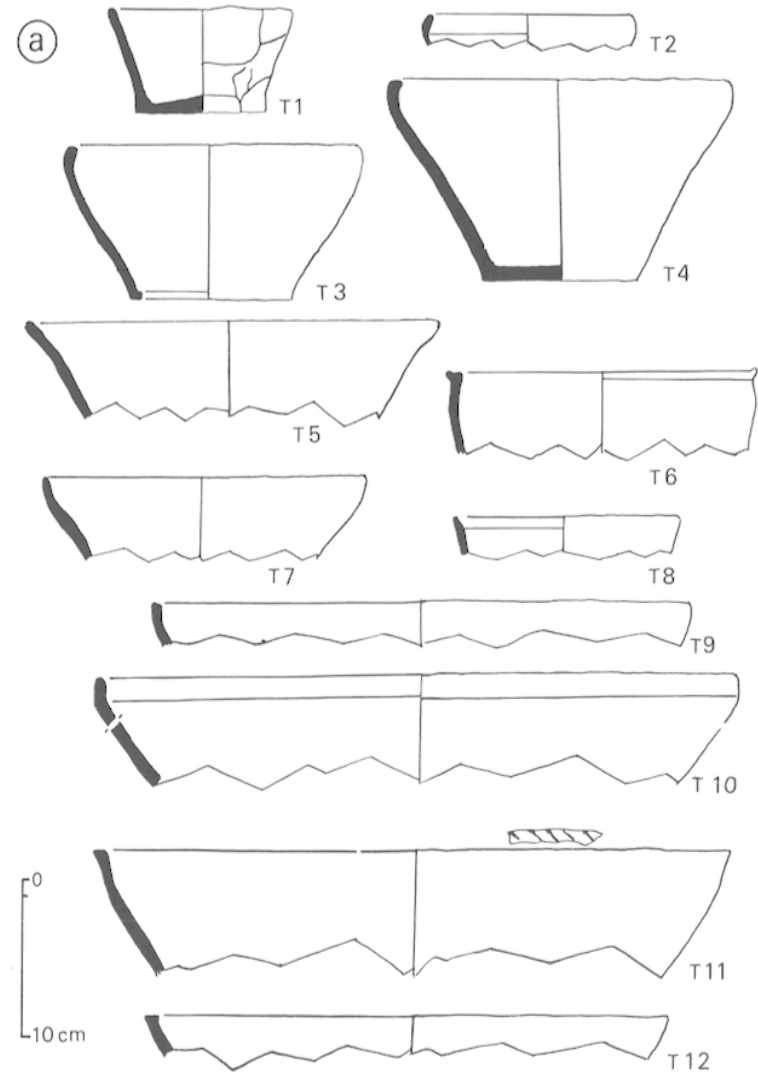

Ce groupe de céramique commune représente $41 \%$ de la céramique indigène. Il est subdivisé en dix-neuf types : douze jattes au sens strict, trois écuelles, une assiette et trois vases à boire. Ces genres de récipients sont utilisés principalement pour le service de la table et pour la préparation culinaire ${ }^{4}$.

- $\mathrm{A} 1$ - Les jattes $\mathrm{T} 1$ à T12 (fig. 3 ), au sens strict du terme, représentent $77 \%$ de la catégorie. Ce sont des formes basses et ouvertes, très évasées, munies d'un rebord plutôt que d'un véritable col. Les formes sont élémentaires, toujours dépourvues de carène. Les dimensions varient : la plus petite des jattes $\mathrm{T} 1 \mathrm{mesure} 7 \mathrm{~cm}$ de hauteur sur $13,5 \mathrm{~cm}$ de diamètre, les grandes jattes ont jusqu'à $40 \mathrm{~cm}$ de diamètre et sont plus évasées. Les lèvres de quelques exemplaires présentent des moulurations qui pourraient suggérer l'utilisation de certains récipients comme couvercles. C'est en particulier le cas dans des formes de type 1,6 et 12 , à rapprocher des formes de couvercles 2, 1 et 4 de La Butte de Sion (Meurthe-et-Moselle). Le répertoire des sous-types 6

4 Les fouilles de 1981 ont permis la mise au jour d'une faisselle constituee d'une jatte A1, sous-type 3 percé de trous.
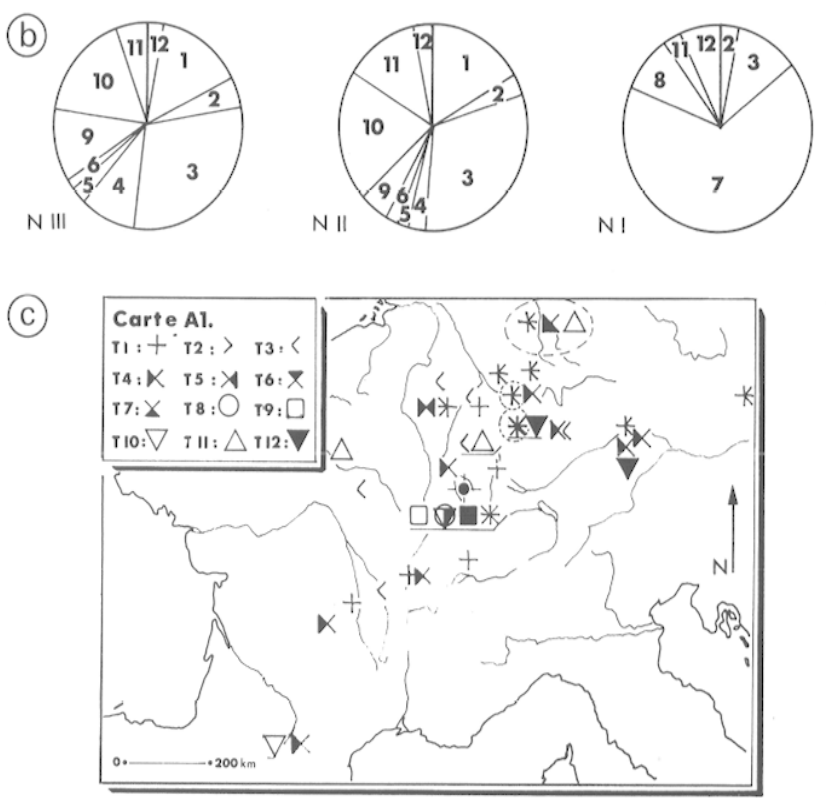

Fig. 3 - Jattes, catégorie A1. a, types 1 à $12 ; b$, répartition des types par niveaux; $c$, diffusion des types dans les sites de référence.

et 12 présents aux niveaux III et II, s'enrichit à cet horizon de la forme 1 . Seuls subsistent au niveau I des sous-types de la forme 12.

- A2 - Les écuelles T13 à T15 (fig. 4) ont une forme comparable aux jattes, mais elles sont plus basses et possèdent un diamètre d'une vingtaine de centimètres.

Les assiettes T16 sont de simples galettes de terre munies d'un rebord plus ou moins élaboré. Le plat, type 16 bis, a la forme d'une grande assiette de $6,5 \mathrm{~cm}$ de hauteur sur $30,5 \mathrm{~cm}$ de diamètre.

- A3 - Les vases à boire T17 à T19 (fig. 5), $9 \%$ de la catégorie, sont répartis en trois types bien différenciés : un gobelet T17, un hanap T18 et un bol T19.

Catégorie $B$ : les coupes (fig. 6 )

C'est une catégorie très homogène, de céramique fine, destinée au service de la table pour contenir boisson et nourriture. Elle représente $8 \%$ du total des vases. Ce sont de petits récipients aux lignes arrondies, de forme basse et ouverte, presque toujours carénées, répartis en neuf types. Les dimensions varient peu : les plus faibles diamètres d'ouverture sont de $13,5 \mathrm{~cm}$ environ (T9), les plus 


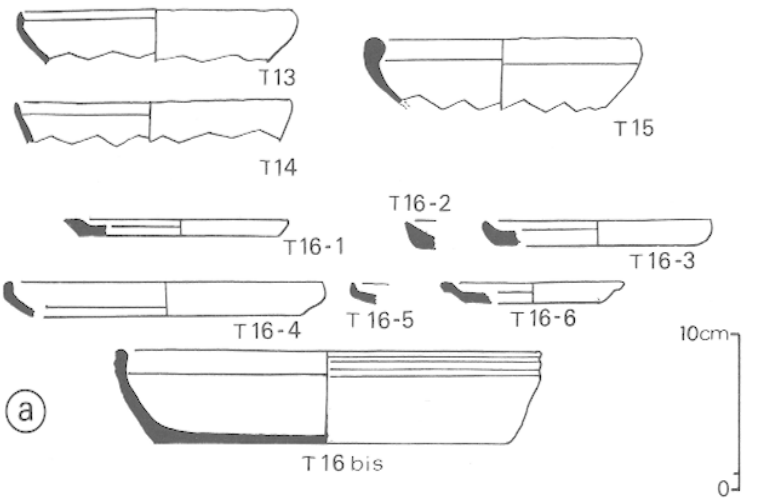

Fig. 4 - Assiettes et écuelles, catégorie A2. a, types 13 à 16 ; $\mathrm{b}$, répartition des types par niveaux; $c$, diffusion des types dans les sites de référence.
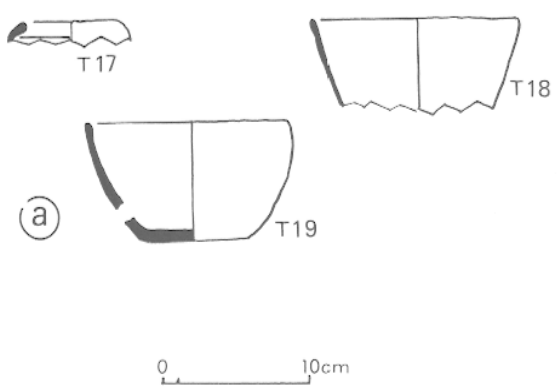

Fig. 5 - Vases à boire, catégorie A3. a, types 17 à 19 ; $\mathrm{b}$, répartition des types par niveaux; c, diffusion des types dans les sites de référence.

larges font $27,4 \mathrm{~cm}(\mathrm{~T} 4)$. La plupart de ces récipients sont apodes.

Catégorie $C$ : les bouteilles (fig. 7)

Ces récipients représentent $12 \%$ de l'ensemble. Il s'agit d'une catégorie de poterie fine, destinée à contenir des liquides, sans doute utilisée pour le service de la table. La nécessité d'une bonne ètanchéité explique au moins en partie le soin accordé à la fabrication de ces vases, autant que le souci esthétique.

Cette catégorie est divisée en douze types bien différenciés. Ce sont des formes hautes et fermées,
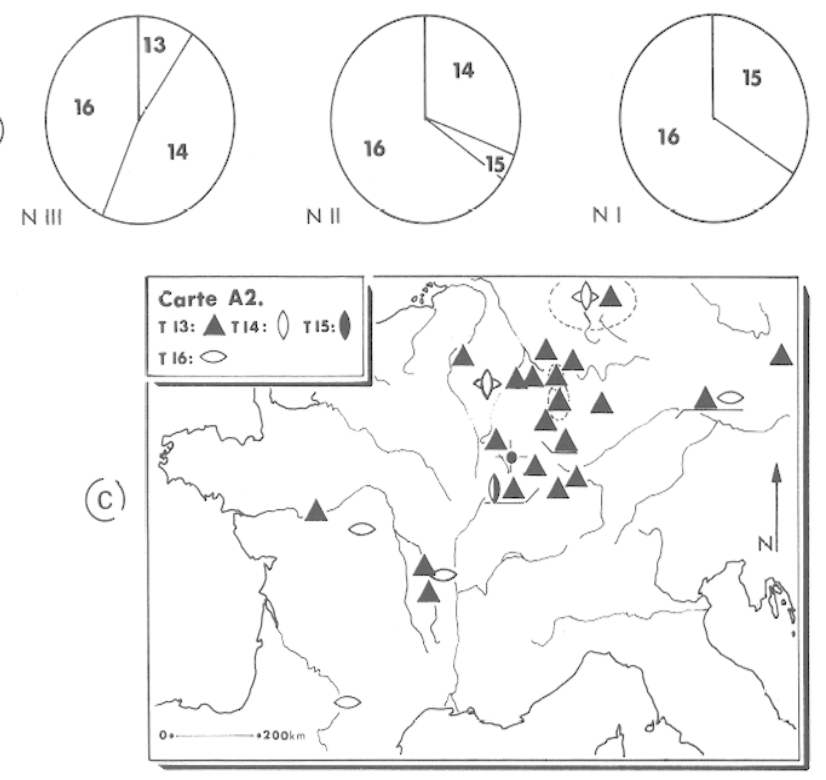

(b)
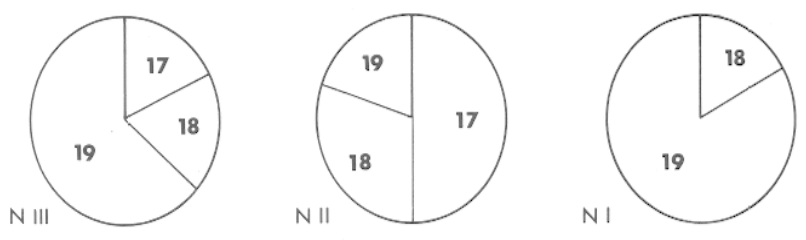

(C)

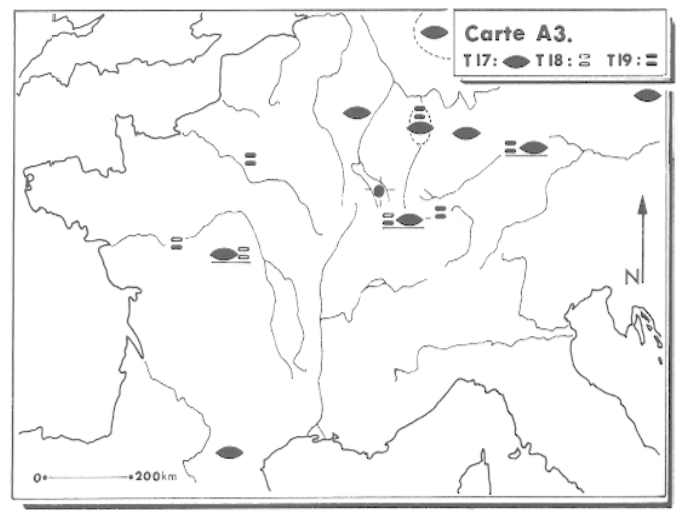

munies d'un col conséquent, les dimensions sont relativement importantes et plus variées que pour les catégories précédentes: les diamètres d'ouverture vont de $9 \mathrm{~cm}$ à $20 \mathrm{~cm}$, la hauteur atteint $30 \mathrm{~cm}$.

Catégorie $D$ : les marmites (fig. 8 )

Cette catégorie de céramique commune, destinée à la coction des aliments est divisée en treize types représentant $19 \%$ au total.

Ces vases ont des formes hautes et fermées d'ovoïdes trapus, assez lourdes. Le diamètre d'ouverture varie de $12,5 \mathrm{~cm}(\mathrm{~T} 7)$ à $24 \mathrm{~cm}$ (T12), compris 

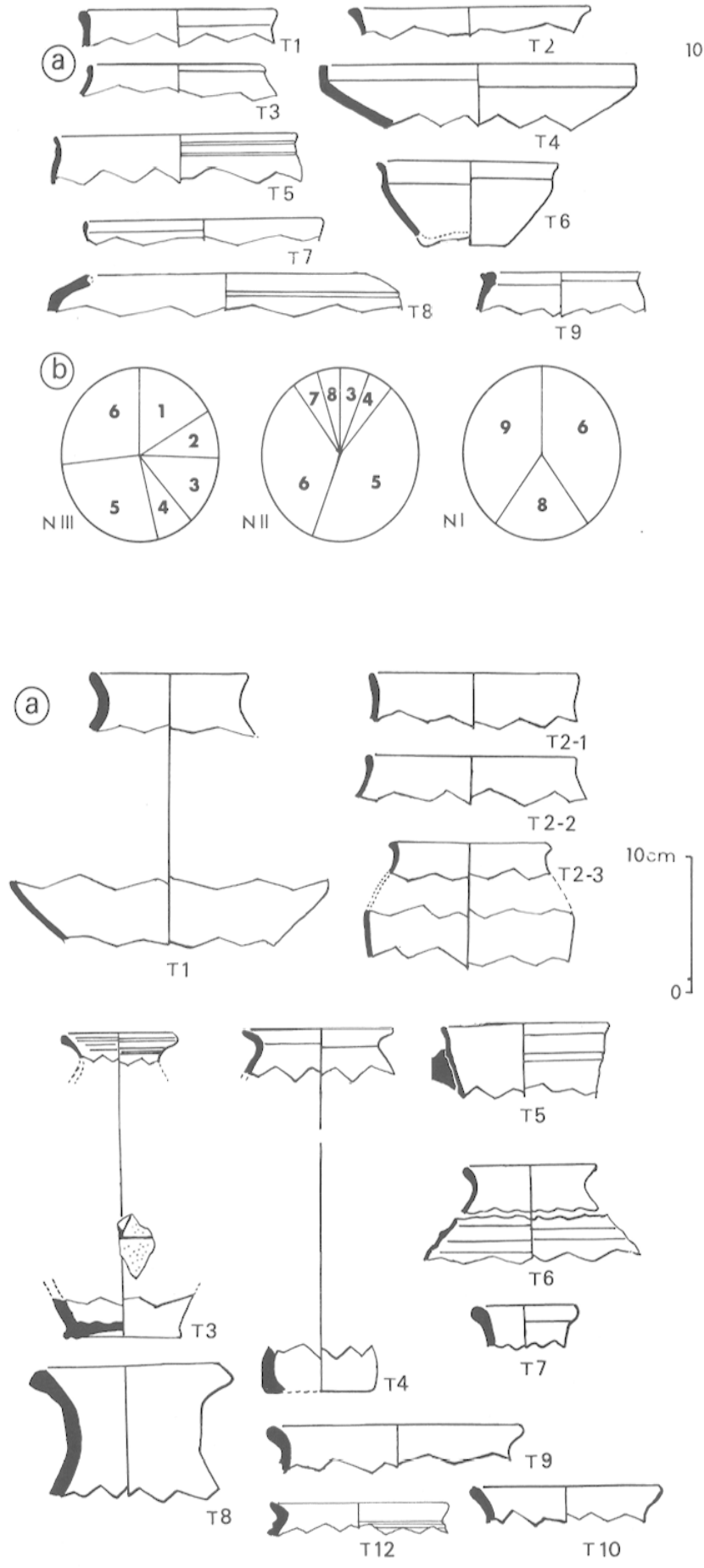

(b)
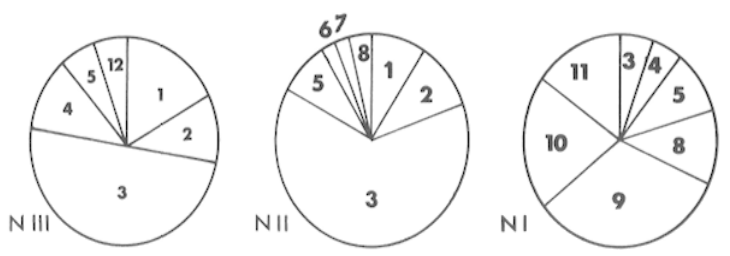

Fig. 6 - Coupes, catégorie B. a, types 1 à $9 ; \mathrm{b}$, répartition des types par niveaux; $c$, diffusion des types dans les sites de référence.

Fig. 7 - Bouteilles, catégorie C. a, types 1 à $12 ;$ b, répartition des types par niveaux; c, diffusion des types dans les sites de référence. (Le type 11 n'est pas représentable.)

généralement entre $15 \mathrm{~cm}$ et $20 \mathrm{~cm}$; il est un peu plus petit que le diamètre maximum qui se trouve presque toujours atteint au niveau de l'épaule du vase. Les parois ont un profil général en $\mathrm{S}$. La hauteur de ces vases varie de $20 \mathrm{~cm}$ à $35 \mathrm{~cm}$ environ.
L'ouverture est munie d'un col court, souvent incurvé vers l'extérieur. Le fond est plat: les marmites devaient donc être posées sur le foyer et non suspendues. Cependant certains exemplaires, peu nombreux, sont dotés de trous de suspension. 


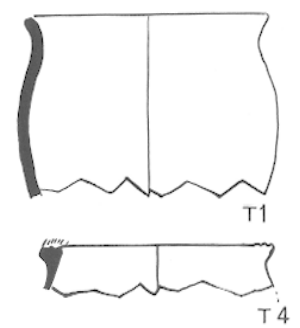

(a)
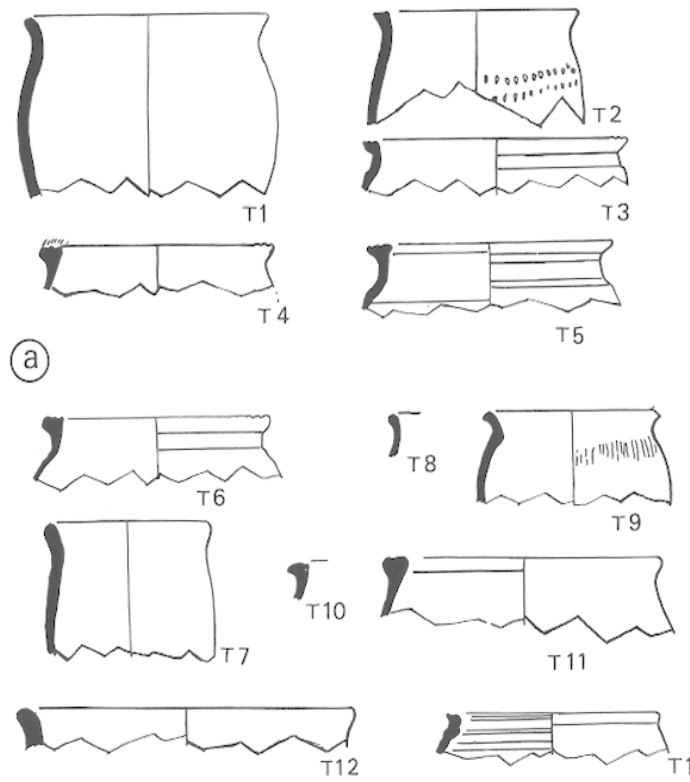

T5

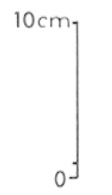

(C)
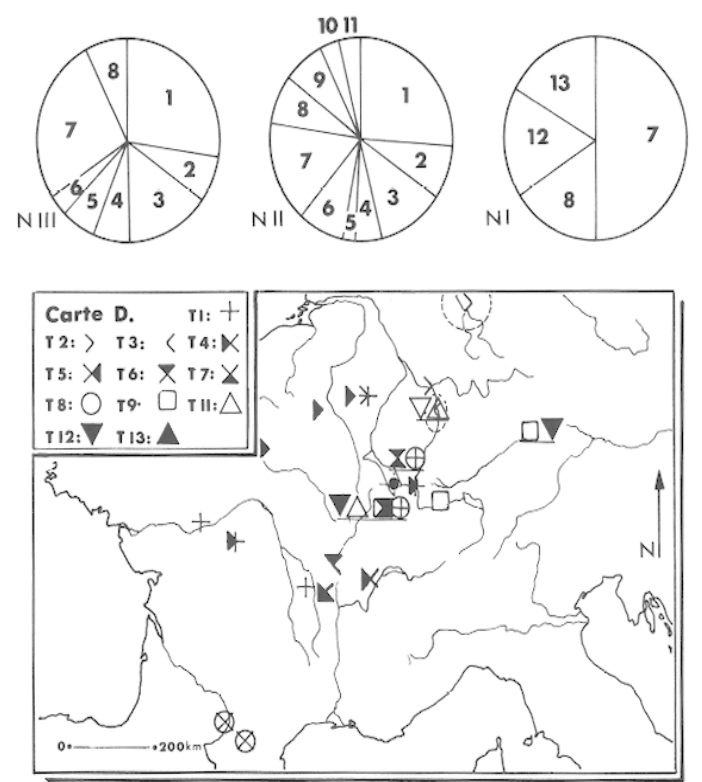

Fig. 8 - Marmittes, catégorie D. a, types 1 à $13 ; \mathrm{b}$, répartition des types par niveaux; $c$, diffusion des types dans les sites de référence.

Catégorie $E$ : les pots (fig. 9)

Ils constituent $12 \%$ du total. Aux niveaux III et II, il s'agit d'une céramique commune; au niveau I, les pots appartiennent à une production fine. Ces vases paraissent avoir été destinés au stockage des denrées, sans que l'on connaisse plus précisément leur usage, il en existe onze types.

Les formes des pots ressemblent beaucoup à celle des marmites : il s'agit de formes hautes (sauf T3) et fermées. Les galbes sont cependant plus variés et parfois plus élégants (T1). Le diamètre d'ouverture de ces vases varie de $13,5 \mathrm{~cm}$ (T5) à $26,4 \mathrm{~cm}$ (T4), la hauteur est comprise entre une vingtaine et une trentaine de centimètres.

Catégorie $F^{\prime}$ : les tonneaux (fig. 10)

Ils représentent $6 \%$ de l'ensemble. Il s'agit d'une catégorie de céramique fine utilisée peut-être pour le service des liquides, répartie en sept types.

Ce sont des formes hautes et fermées, à la silhouette ovoïde parfois très élégante. L'ouverture est munie d'un rebord ou d'un col embryonnaire. Certains vases sont de petite taille $(\mathrm{T} 2: 5,8 \mathrm{~cm}$ de diamètre à l'ouverture; $19,5 \mathrm{~cm}$ de hauteur) d'autres beaucoup plus volumineux $(21 \mathrm{~cm}$ de diamètre d'ouverture et une hauteur estimée à $75 \mathrm{~cm}$ pour le type 1).
Catégorie $G$ : les dolia (fig. 10,d)

Ces vases sont peu fréquents $(1 \%)$. Il s'agit d'une imitation, c'est-à-dire d'une forme d'origine italienne adoptée et fabriquée en Gaule, qui doit être rangée dans la céramique commune. Le volume de ces vases les rend difficilement transportables, quoique les dolia trouvés à La Pierre d'Appel soient relativement petits : il existe ailleurs en Gaule des dolia de la taille d'un homme. Il s'agit incontestablement de récipients de stockage, peut-être utilisés comme silos à grains. Cette catégorie est subdivisée en un protolype et deux types.

Ces vases ont une forme haute et fermée, assez grossière. La panse est quasi cylindrique: elle s'achève vers le haut en une calotte hémisphérique et se rètrécit vers le bas. L'ouverture est assez large, munie d'un col court et épais : les diamètres d'ouverture varient de 30 à plus de $40 \mathrm{~cm}$, la hauteur de ces vases pouvait atteindre le mètre.

A titre de comparaison, il est possible de signaler l'existence d'importations italiques (fig. 11) réduites à six vases : quatre campaniennes et deux amphores. La céramique campanienne se compose d'un récipient de la série $A$, forme 36 , d'une coupe de la série $B$, forme $1 b$, d'une poterie de la forme A5 ou B5, enfin d'un fragment de carène indéterminable. Les deux amphores sont des amphores vinaires républicaines appartenant respectivement aux types Dressel 1A et Dressel 1B. 

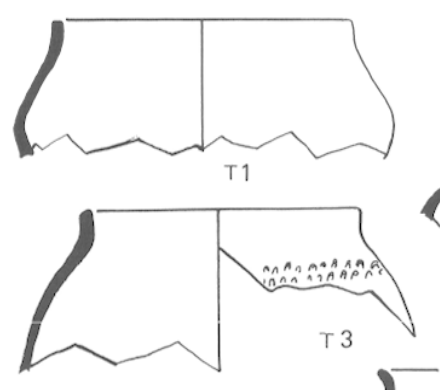

(a)
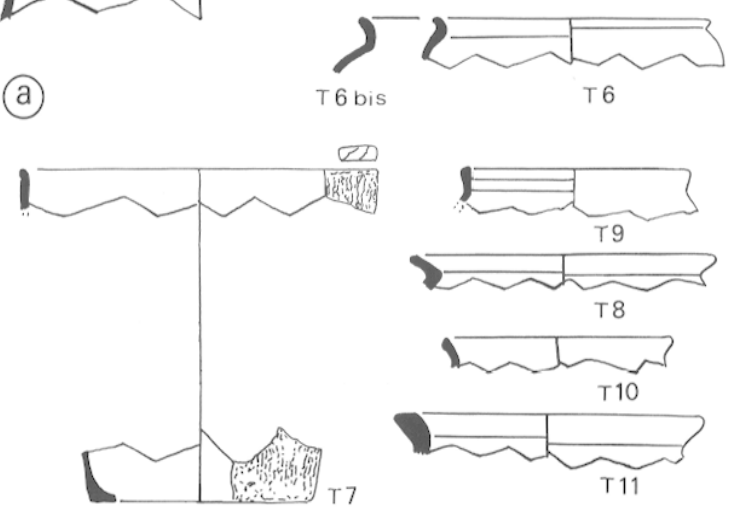

(b)
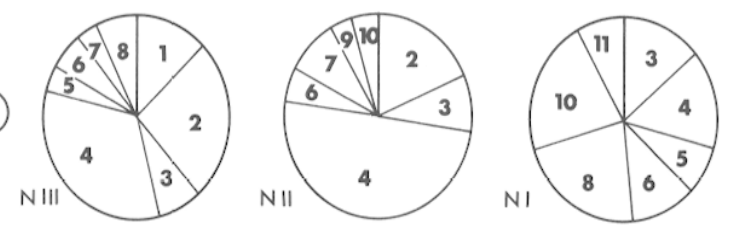

${ }_{0}^{10 \mathrm{~cm}}$

(C)

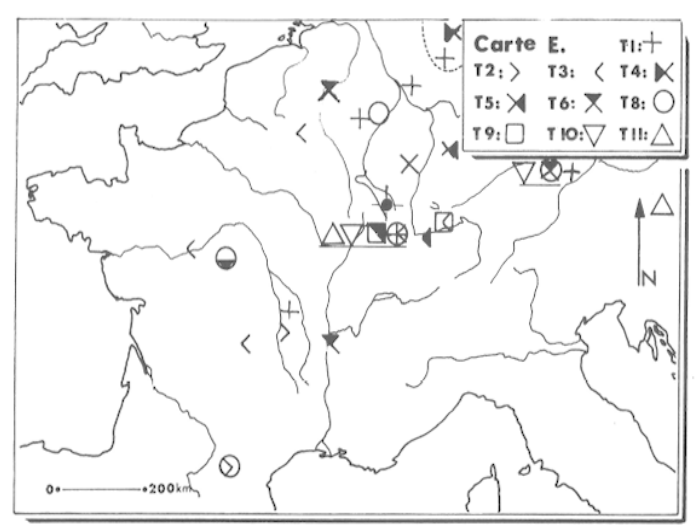

Fig. 9 - Pots, catégorie E. a, types 1 à 11 ; b, répartition des types par niveaux; c, diffusion des types dans les sites de référence.

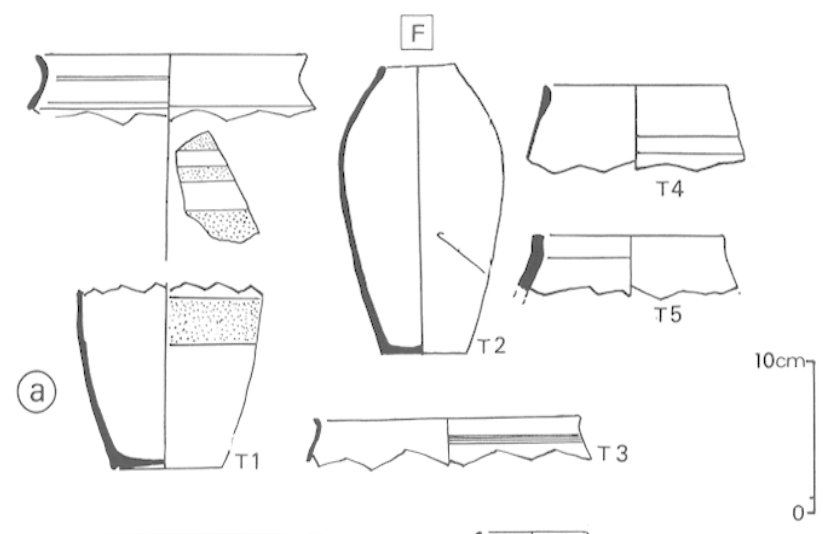

(C)
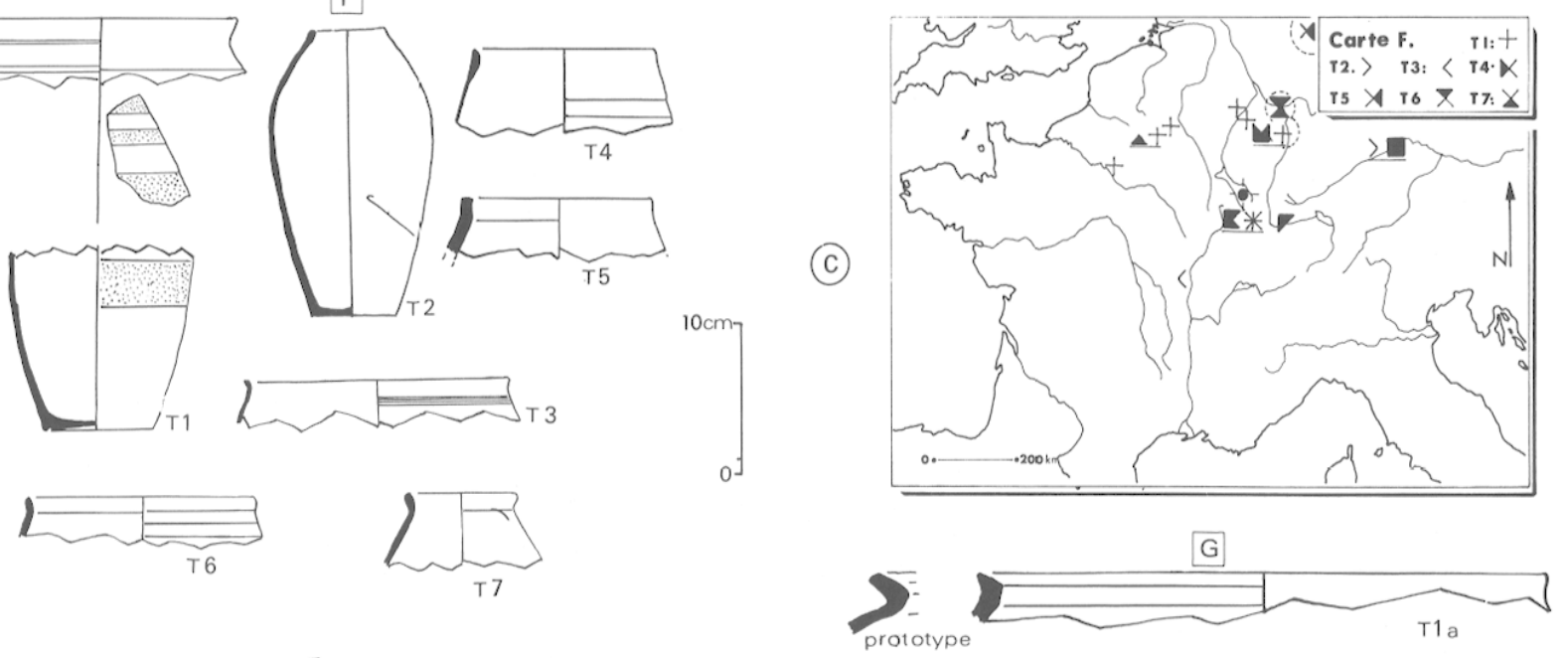

(b)

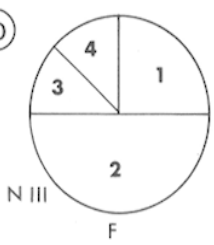

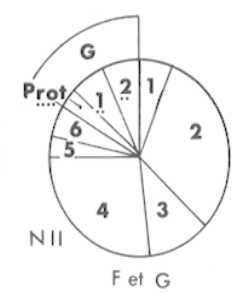
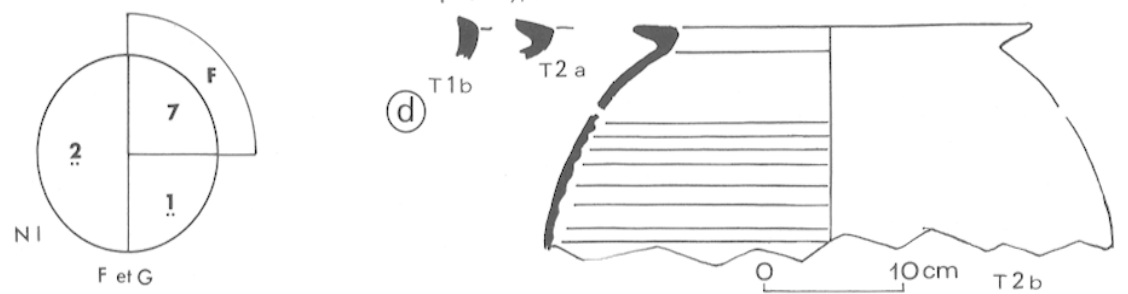

Fig. $10-a$, tonneaux, catégorie $F$. a, types 1 à $7 ; b$, répartition des types des formes $F$ et $G$ par niveaux; $c$, diffusion des types de $\mathrm{F}$ dans les sites de référence; d, dolia, catégorie $\mathrm{G}$, prototype et types 1 et 2 . 


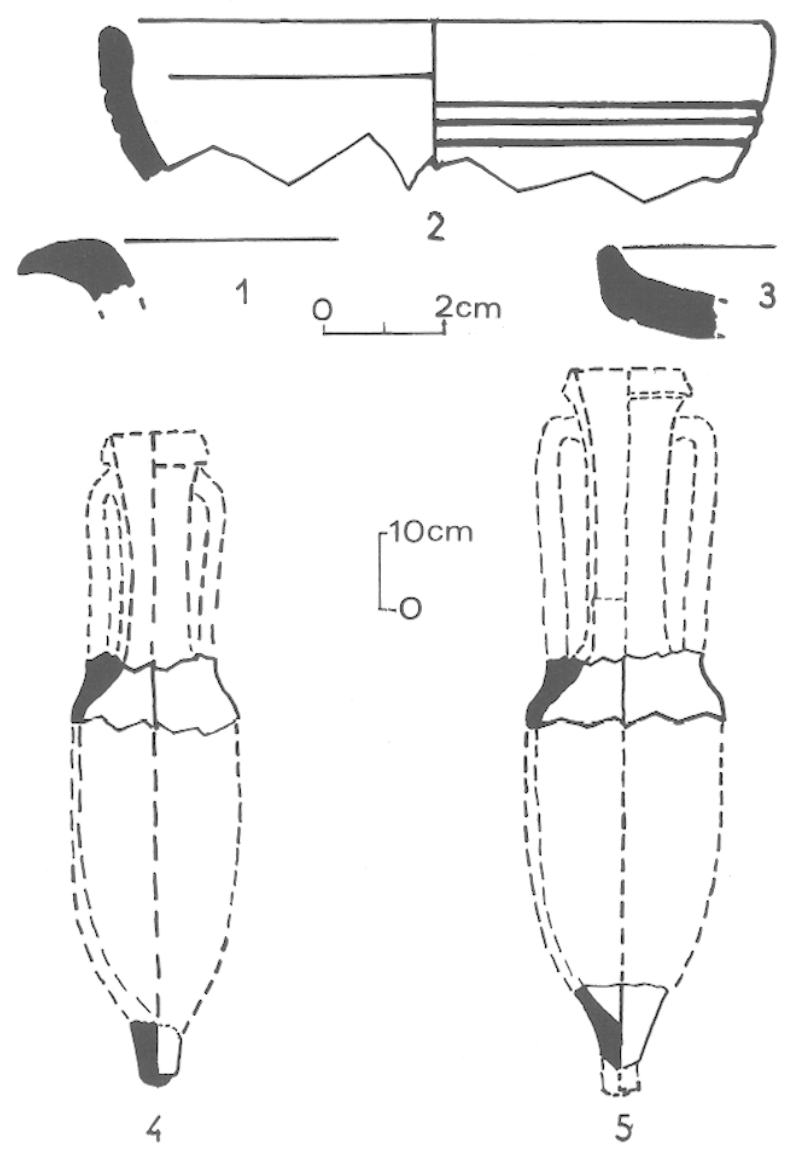

Fig. 11 - Céramiques d'importation (1 à 3 ) et amphores italiques (4 et 5$)$.

\section{Comptages : STABILITÉ ET RENOUVELlement}

Outre la classification, cet ensemble de 605 vases a été l'objet de calculs statistiques élémentaires : ainsi 217 vases $(36 \%)$ proviennent du niveau III, $292(48 \%)$ du niveau II et $96(16 \%)$ du niveau I. Ces simples chiffres donnent une première idée de l'évolution générale : en effet, si l'on tient compte de la durée des occupations successives entre 90 et 170 ans pour le niveau III, 20-30 pour le niveau II, 40 pour le niveau $\mathrm{I}$, le dynamisme et l'expansion du niveau II sont tout aussi manifestes que la régression du niveau I.

Les 605 vases sont donc répartis en sept catégories subdivisées en 73 types. Quantitativement, la répartition des types est inégale à l'intérieur des catégories. A chaque niveau, un ou deux types seulement constituent l'essentiel de l'effectif considéré, les autres, parfois nombreux, restent secondaires. Les types principaux existent souvent dans les trois niveaux : on en compte au total quinze qui sont permanents ( $21 \%$ des types) et représentent 246 vases $(41 \%)$ : quantité très importante. En contrepartie, il existe 24 types ( $33 \%$ ) spécifiques, c'est-à-dire propres à un seul niveau, qui regroupent 78 vases $(13 \%)$.

Le phénomène de spécificité varie selon les niveaux : aux niveaux les plus anciens, les types spécifiques sont marginaux, reflets d'influences faibles ou lointaines, traduisant une certaine ouverture économique et culturelle, ou bien tentatives timides de nouvelles formes. Au niveau I, il traduit un profond renouvellement puisque le mobilier constitué par de nouveaux types représente plus de la moitie des vases.

Les formes de la vaisselle non céramique ont sans doute influencé l'évolution de la céramique. Toutefois, des facteurs économiques ou des faits de société (modification des habitudes alimentaires à partir du niveau II sous l'effet des influences méridionales) ont certainement eu une importance considérable.

\section{INFLUENCES}

Enfin, ce mobilier a été comparé avec celui décrit dans diverses publications archéologiques: 62 sites (fig. 1) dont le mobilier présentait des analogics plus ou moins importantes ont été ainsi recensés, ce qui permet quelques réflexions sur les différentes influences et leur évolution (fig. $3 \mathrm{c}$ à $10 \mathrm{c}$ ). Pour la commodité de l'étude, nous considérerons les sites en trois sous-ensembles : un domaine rhénan (le bassin hydrographique rhénan), un domaine oriental qui regroupe les sites allemands à l'Est du Rhin et les sites tchécoslovaques, et un domaine occidental avec les sites de territoires aujourd'hui francophones.

Incontestablement, les liens sont très étroits entre La Pierre d'Appel et l'ensemble du domaine rhénan: trois catégories et la plupart des types semblent caractéristiques de cette région. Les relations sont privilégiées avec Bâle qui, centre important et bien situé, paraît particulièrement apte à rayonner sur cette région. Les rapports ont sans doute aussi été nombreux avec les régions plus en aval du fleuve: le Palatinat et, plus au nord, la Hesse. Vers l'est, il est probable que de nombreux contacts existaient, peut être par l'intermédiaire de villes comme Bâle : il existe de nombreuses analogies avec les sites de Manching et même les oppida de Tchécoslovaquie. Dans le domaine occidental, les relations ont dû être abondantes mais aussi plus diffuses : des rapports certains existent avec des sites 
aussi éloignés dans le Sud-Ouest, que Toulouse ou La Lagaste (Aude) ${ }^{5}$.

Chronologiquement, ces influences se répartissent en deux phases : la première correspond, aux niveaux III et II, à une période d'ouverture, de relations nombreuses avec des régions parfois très éloignées. En revanche, au niveau I, la régression est nette et brutale : ce niveau est amputé de ce qui caractérisait l'influence occidentale et cela se traduit par un repli sur le domaine rhénan.

\section{LA DÉCORATION}

\section{Classification}

Le phénomène de la décoration joue un rôle important à La Pierre d'Appel puisque 187 vases $(31 \%)$ ont été décorés. Les différents décors ont été classés en 6 types. Cette classification est essentiellement fondée sur la technique du décor (fig. 12).

Le type 1 regroupe tous les décors estampés ou incisés à l'aide d'un instrument simple : poinçon, peigne, balayette ... il s'agit donc de décors en creux, souvent appliqués rapidement et principalement utilisés pour la céramique commune. Le type 1 est subdivisé en 7 sous-types selon l'outil employé et le motif du décor.

Le sous-type 1-a recouvre les décors estampés aux motifs de disque ou de croissant. Ces deux motifs sont considérés comme des symboles astraux : le croissant est probablement un symbole lunaire, et le disque un symbole solaire. La position des croissants n'est pas fixe : ils peuvent être tournés vers la droite ou vers la gauche, le haut ou le bas. Les poinçons utilisés ont dû être façonnés à dessein.

Le sous-type 1-b désigne les motifs estampés en forme de goutte d'eau ou de grain de blé, oblongs et arrondis. Ils n'ont pas de position fixe. Ce sous-type est probablement obtenu par l'application d'une baguette à l'extrémité arrondie, la forme plus ou moins ovale du motif provenant de l'angle selon lequel cette baguette s'enfonce dans la paroi du vase.

Le sous-type 1-c rassemble les autres décors estampés, il se compose essentiellement de formes géométriques: triangles, petits carrés; on y a également rangé deux motifs moins fréquents : des cercles imparfaits et un motif de forme indéterminable. Sauf dans ce dernier cas, il s'agit probablement

5 Voir en annexe la bibliographie complémentaire qui énumère les sources de la figure 1 . de poinçons taillés pour cet usage. Le motif est franchement imprimé dans la pâte.

Le sous-type 1-d regroupe les décors incisés obtenus à l'aide d'un peigne, d'un pinceau ou d'une balayette. Ces trois outils laissent des traces formées de lignes parallèles, mais selon le coup de main, des effets différents peuvent être produits. Le peigne a été utilisé de façon simple pour dessiner des stries verticales ou des bandes horizontales faisant le tour du vase. Une variante souvent employée consiste à tracer une bande non plus droite mais ondoyante. D'autre part, la panse de certains vases a été entièrement peignée sans souci de tracer des motifs décoratifs, même sommaires : il s'agit alors plutôt d'un effet de texture. Cette dernière méthode permet de répartir la pâte plus régulièrement sur la surface du vasc, cffaçant les colombins trop apparents et produit une surface rugueuse qui facilite la préhension.

Le sous-type 1-e est également tracé à l'aide d'un peigne, utilisé non pour inciser mais pour estamper, le motif apparait sous la forme de petits creux arrondis, généralement disposés par bande de trois ou quatre lignes.

Le sous-type 1-f comprend les décors incisés en forme de traits droits ou obliques ou en croix de Saint-André. Ce dernier, représenté par deux exemplaires, existe uniquement au niveau I. Ces motifs sont disposés parallèlement, formant une bande continue autour du vase. L'outil utilisé n'est qu'une simple baguette et ce décor ne présente pas le parallélisme $\dot{d u}$ sous-type $1-d$. Les lignes incisées sont plus larges et plus écartées. La longueur des traits et l'angle selon lequel est appliquée la baguette varie d'un vase à l'autre.

Le sous-type $1-g$ représente les décors de cannelures et les lignes horizontales incisées, semblables par la technique aux traits du sous-type 1-f.

Les motifs du type 2 sont constitués d'une protubérance de forme plus ou moins conique enchâssés dans un creux ovale, comme un œil dans une orbite. Ce décor oculè est appliqué à l'aide d'un poinçon spécial et disposé en bandes de trois ou quatre lignes d' "yeux» disposés à intervalles réguliers. Ce type de décor, rare à La Pierre d'Appel, date de la période gallo-romaine précoce (niveau I) et a été utilisé par des ateliers spécialisés de production semi-industrielle.

Type 3 : il s'agit de décors estampés au moyen d'un sceau cylindrique (ou roulette) que l'on fait rouler tout autour du vase afin d'obtenir une bande de motifs régulièrement imprimés. Les motifs appliqués sont de deux sortes: d'une part des motifs 


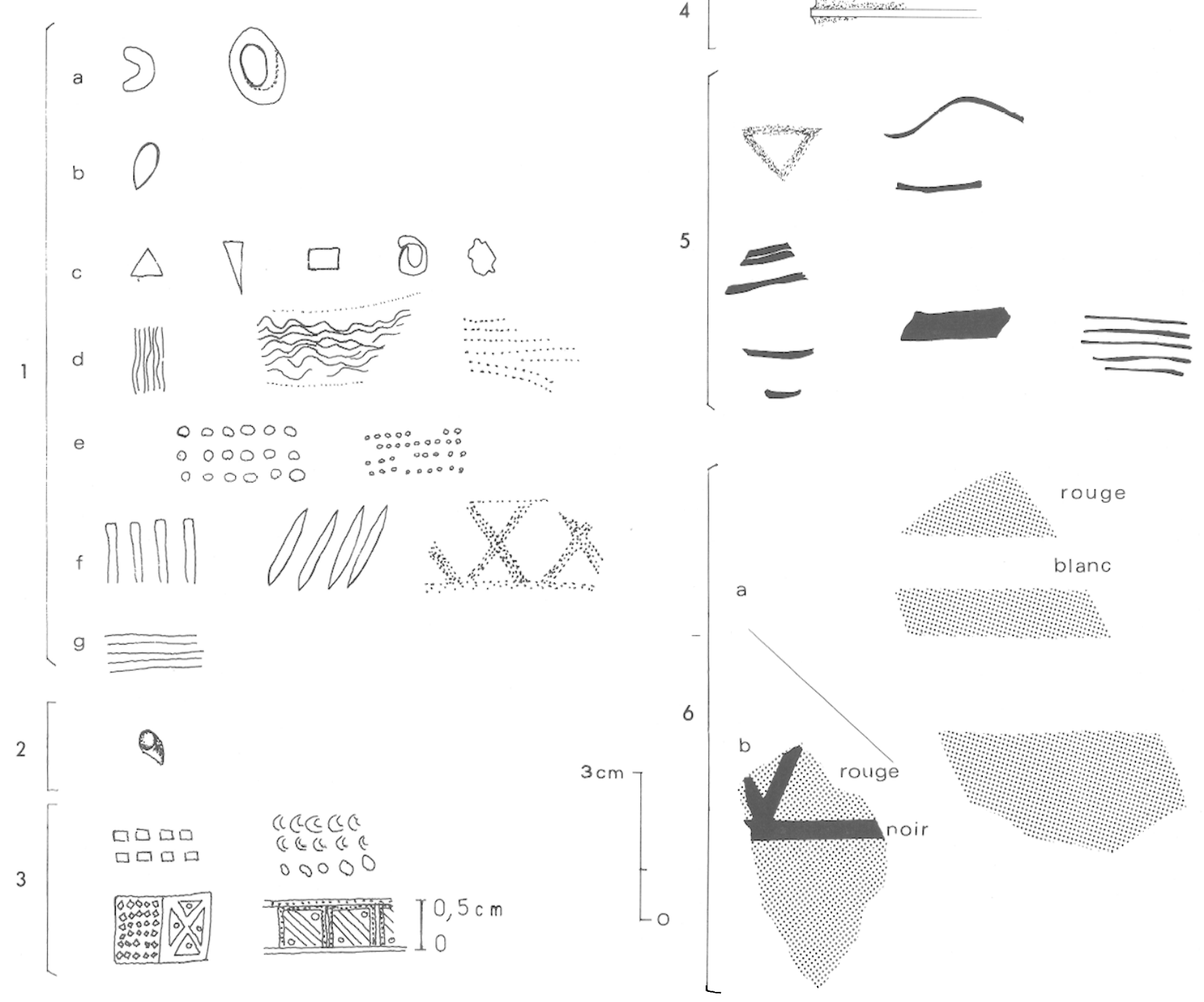

Fig. 12 - Typologie des décors : types 1 à 6 .

simples empruntés aux décors de sous-types 1- $a$ et 1-c, d'autre part des motifs fins, beaucoup plus recherchés, d'un travail très soigné.

Le type 4 regroupe les décors en baguette (moulure en surépaisseur tournée dans la masse).

Le type 5, assez rare à La Pierre d'Appel, comprend les motifs obtenus à partir du lissage et du graphitage.

Type 6 : la céramique peinte. A La Pierre d'Appel, elle est présente sous deux variantes : d'une part, une céramique à bandes rouges et blanches (6a), qui est typique de la fin de l'Indépendance et du début de la Conquête romaine; d'autre part une céramique à dessins sombres sur fond clair ou crème (6b) mais qui ne peut être bien étudièe faute de fragments de taille suffisante. La plupart des vases peints sont de formes hautes et fermées, ce qui rattache cette céramique au groupe occidental distingué par F. Maier ${ }^{6}$.

Correspondance décor-Forme. Évolution

Ces décors ne sont pas indifféremment employés sur les diverses catégories de formes: il existe des correspondances obéissant à des raisons pratiques, techniques et esthétiques. La céramique commune est essentiellement décorée par incision ou estampage: les formes basses et ouvertes (jattes) sont

6 F. Maier, Zur Bemalten Spätlatenenkeramik in Mitteleuropa, Germania, 41, 1963, p. 259-268. 

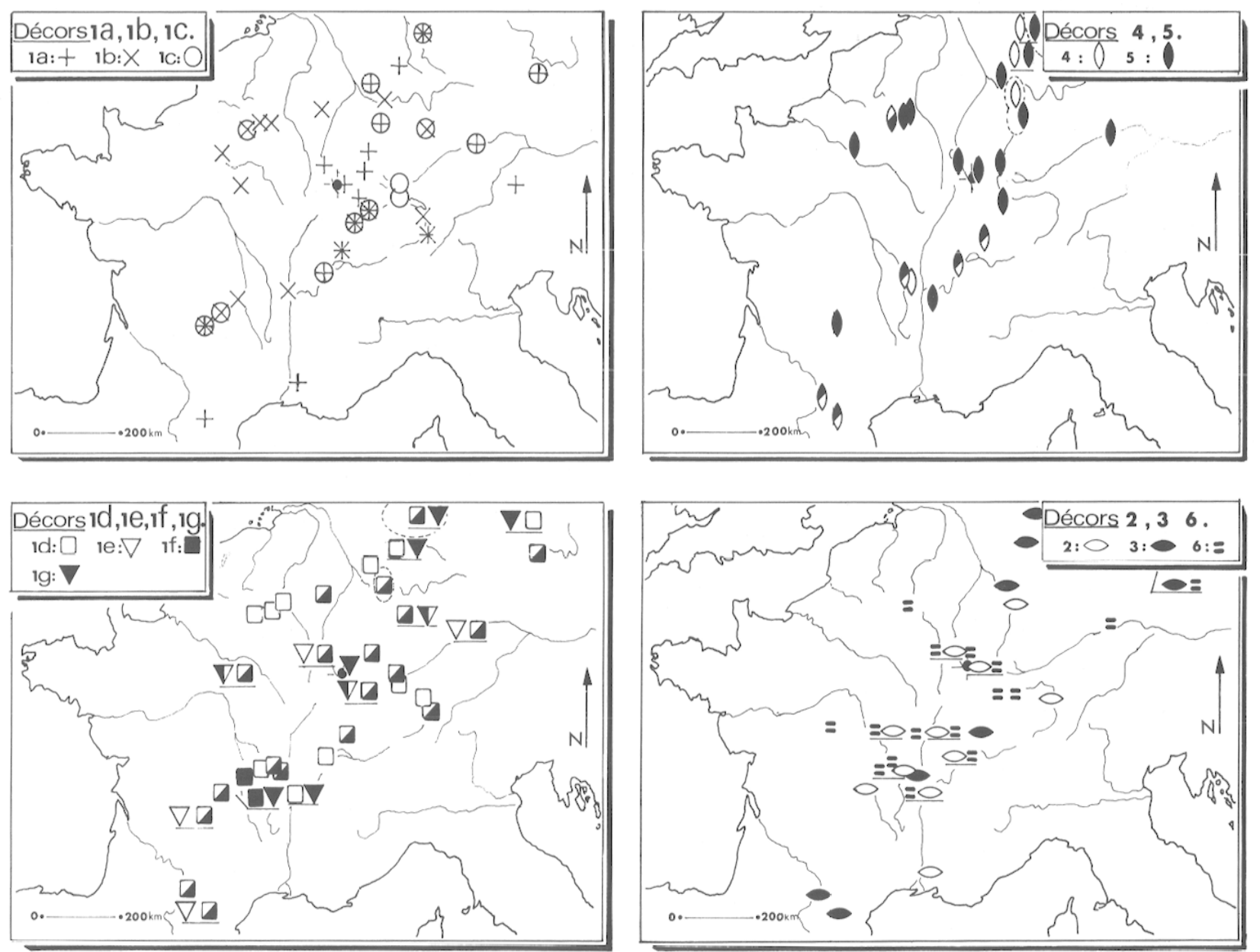

Fig. 13 - Diffusion des décors dans les sites de référence.

rarement décorées tandis que les formes hautes (marmites et pots) le sont plus souvent. La céramique fine a souvent reçu un décor peint (coupes, bouteilles, tonneaux). En outre, les bouteilles sont souvent décorées de lignes horizontales, droites ou ondées, quelle que soit la technique employée (1-d, 1$g, 4,5,6)$. En revanche, ce sont surtout des pots qui ont reçu des décors de types 3 (roulette) et 2 . Ainsi l'évolution quantitative des décors est-elle conditionnée par celle des formes. Qualitativement, nous remarquons cependant que les types 2,3 et 4 , qui traduisent tous une certaine sophistication technique, représentent $21 \%$ des décors du niveau I.

L'évolution du type 6 présente un cas particulier car elle doit être envisagée dans le cadre de l'évolution comparée de deux sortes de céramique fine : la poterie peinte et la "grise lissée" (imitation gauloise des céramiques italiques à vernis noir).
Progressivement, la première supplante la seconde avec les pourcentages respectifs suivants:

- $22 \%$ et $78 \%$ au niveau III;

- $54 \%$ et $46 \%$ au niveau II;

- $67 \%$ et $33 \%$ au niveau $\mathrm{I}$.

Les comparaisons avec d'autres sites (fig. 13) posent plus de problèmes que pour l'étude des formes, cependant elles permettent de constater l'existence de deux sortes de décors: des décors traditionnels, très anciens sans doute, et largement diffusés (types $1,4,5$ ) donc peu caractéristiques, et des décors plus récents, produits d'amélioration techniques (types $2,3,6$ ) qui semblent provenir du Centre-Est de la Gaule. Le domaine rhénan et La Pierre d'Appel en particulier paraissent peu novateurs en ce domaine. 
$\mathrm{Au}$ terme de cette étude, il convient maintenant d'essayer de replacer les données archéologiques observées dans leur contexte chronologique et culturel. Nous pouvons employer deux solutions : d'une part, celle qui tient aux capacités chronologiques du site proprement dit, d'autre part, recourir aux méthodes d'analyses des données.

Dans le premier cas, il est indéniable que La Pierre d'Appel présente des caractéristiques assez remarquables touchant à la fois à la stratigraphie et à quelques ensembles clos. En ce qui concerne la stratigraphie, il faut souligner la mise en évidence de cinq niveaux d'occupation et trois de destruction en connexion avec trois systèmes fortifiés barrant l'éperon. Les sépultures présentent un cas différent : les recoupements entre tombes ou entre celles-ci et les structures d'habitat d'une part, les constructions funéraires appuyées les unes sur les autres, d'autre part, permettent des classements limités mais complémentaires des précédents. C'est l'accumulation des données élémentaires que sont les pourcentages de formes à l'intérieur d'une même couche et à travers les couches qui permet de reconnaître deux phases de faciès régionaux. Ceux-ci procèdent pour l'essentiel de la civilisation celtique d'Allemagne du Sud, et plus précisément de la culture dite du Rhin supérieur. C'est ainsi que les vestiges des niveaux IV/III2 à II2, attribuables à La Tène D1, sont comparables à ceux des grands habitats rhénans tels que Bâle-Gasfabrik ${ }^{7}$ et Breisach-Hochstetten ${ }^{8}$. Par ailleurs, certains vestiges issus du niveau IV/III2, revêtent des caractères plus anciens, attribuables à La Tène C2. Ils partagent des traits communs avec du matériel découvert dans le pays de Trèves, le Palatinat $^{9}$ et sur la rive droite du Rhin comprise entre Ruhr au nord et Main au sud (région de Hesse $)^{10}$. Quant au mobilier des niveaux III et I, il est attribuable à La Tène D2 ("gallo-romain précoce" de J.-J. Hatt) et peut être rapproché de celui

7 A. Furger-Guntr, L. Berger, Katalog und Tafeln der Funde aus der Spätkeltischen Siedlung Basel-Gasfabrik, Baslet Beitrage zur Ur- und Frühgeschichte, 7, Derendingen Solothurn, 1980, 450 p., 175 fig.

8 I. Stork, Die Keltische Siedlung von Breisach-Hochstetten, Archäologische Nachrichten aus Baden, 15, 1975, p. 3-9.

9 H. J. Engers, Die Hallstat und Latenekultur in der Pfalz, Veröffentlichung der Pfälzischen Gesellschaft zur Förderung des Wissenschaften in Speyer, 55, 1967, $122 \mathrm{p}$.

10 H. Behaghel, Die Eisenzeit in Raume des Rechlsheinischen Schiefergebirges, Wiesbaden, 1949, 156 p., 45 pl., 8 cartes. découvert à Bâle-Münsterhügel ${ }^{11}$ et Breisach-Münsterberg ${ }^{12}$. Toutefois, il faut se garder de vouloir rétablir des rapprochements trop étroits, surtout avec des sites lointains, et c'est ici qu'interviennent les méthodes d'analyse.

La méthode typologique (analyse des techniques de fabrication, des formes, des décors), confortée par la stratigraphie, permet de distinguer des types permanents que traversent les générations sans grand changement et des types spécifiques qui n'existent que pendant un bref moment. C'est ainsi qu'une comparaison entre les deux sites de Bâle et celui de La Pierre d'Appel permet de constater que $25 \%$ des formes et le type 2 des décors identifiés sur celui-ci sont inexistants sur ceux-là. En particulier, les décors hessois sont absents de Bâle alors qu'ils existent à La Pierre d'Appel. La comparaison entre ces deux sites met à la fois en évidence les similitudes et les discordances. Ces dernières révèlent la présence de populations d'origines différentes intégrées à la communauté culturelle du Rhin supérieur. Peut-être pouvons-nous différencier à l'intérieur de cette culture un groupe montagnard (vosgien) distinct de celui de la vallée (alsacien-badois)?

Nous avons déjà souligné les difficultés qu'il y avait à faire concorder la chronologie relative avec des datations absolues : $-250-170$ avec une marge de $\pm 100 ;-60$ avec une marge de $\pm 50^{13}$. Sans rouvrir le débat, on se contentera de rappeler ici que les datations proposées, sans recours aux quatre fragments de Campanienne $A$ et $B$ et aux débris de deux amphores Dressel IA et IB, aboutissent au découpage suivant ${ }^{14}$ :

- niveau III : seconde moitié du III s. / premier quart du $\mathrm{II}^{\mathrm{e}}$ s. av. J.-C. à 80 av. J.-C.;

- niveau II : 80 av. J.-C. à la Conquête;

- niveau I : de la Conquête à Auguste, 15 av. J.-C.

11 A. Furger-Guntı, Die Ausgrabungen in Basler Münster I, Die Spätkeltische u. Augusteische Zeit, Basler Beiträge Zur Ur- und Frühgeschichte, 6, Derendingen-Solothurn, 1979, $313 \mathrm{p}$.

12 H. BEndfr, Neuere untersuchungen auf dem Münsterberg in Breisach, 1966-1975, I, Die Vorrömische Zeit, Archaeologische Korrespondensblatt, 6, 1976, p. 213-224.

13 A. Deyber et alii, op. cit., p. 214-215 et fig. 28, p. 213.

14 Sur l'aspect proprement historique, consulter A. Deyber, Les frontières des peuples préromains dans l'Est de la Gaule à la fin de l'époque de La Tène, Caesarodunum, Centre de Recherches A. Piganiol et Institut d'Études latines de l'Université de Tours, 1981, p. 28-44. 
La confrontation avec des sites voisins parait hautement souhaitable pour affiner ce schéma chronologique, et notamment valider l'hypothèse d'une nouvelle périodisation de La Tène finale dans les Vosges moyennes. En l'occurrence, il s'agirait d'une Tène finale en trois phases dont la première serait prise sur La Tène moyenne en chronologie traditionnelle (La Tène C2 du "système germanique s) $)^{15}$.

\section{Marc Dalaut et Alain Deyber}

15 Cette question a été débattue lors de la table ronde Gaule interne et Gaule méditerranéenne aux II et ter siècles avant J.-C. : confronlations chronologiques organisee par le Centre de Recherches Archéologiques du C.N.R.S. à Sophia-Antipolis les 11-12-13 novembre 1986. Voir notamment les rapports de MM. J.-L. Floufst, O. Buchsenschutz et A. Colin, $\Lambda$. Duval dans la prépublication remise aux participants.

N.B. - Nous ne saurions clore ce travail sans rendre hommage à $\mathrm{M}^{\mathrm{me}} \mathrm{E}$. Ladier à qui nous devons l'établissement, en 1973-1974, des premières statistiques complétées depuis par nos soins. Notre reconnaissance va également à MM.G. Delhougne et B. Guillet, MM. les professeurs Jurain et Ch. Janot de l'Université de Nancy I, sans le soutien technique et scientifique desquels ce travail serait resté très imparfait. Enfin, nous avons une grande obligation envers $M^{\text {lle }}$ A. Colin et M. J.-L. Flouest pour les nombreuses remarques, suggestions et critiques que l'examen du dossier leur ont suggérées.

L'ensemble de l'illustration est l'œuvre de Marc Dalaut.
Chaque numéro renvoie à la figure 1 .

1 Étival-Clairefontaine, La Pierre d'Appel (Vosges)

Deyber A., La céramique de La Tène III à l'oppidum de La Pierre d'Appel, commune d'Étival-Clairefontaine (Vosges), Revue Archéologique de l'Est, XXIII, 1972, 1-2, p. 55-76.

Deyber A., Les structures d'habitat laténien des peuplades préromaines du Nord-Est de la Gaule; point des connaissances, problèmes et directions de recherches, L'Habitat à l'Age du Fer en Europe tempérée - l'évolution de l'habitat en Berry. Actes du colloque organisé les 2729 octobre 1978 à Levroux (Indre), Centre interinstitutionnel pour la diffusion des publications en Sciences Humaines, 1981, Paris, p. 89-100, fig. 7.

Deyber A., avec la collaboralion de M. Dalau'T, E. Goaziou-Ladier et A. Weisrock, L'habitat fortifié laténien de La Pierre d'Appel à ÉtivalClairefontaine (Vosges), Gallia, 42, 1984, p. 175217,28 fig., 1 tableau.

2 Achenheim-Oberschaeffolsheim (Bas-Rhin) Hatt J.-J., Schmitt G., Zumstein H., Fonds de cabane à Achenheim-Oberschaeffolsheim (Bas-Rhin), Cahiers Alsaciens d'Archéologie, d'Art et d'Histoire, 6, 1953, p. 55 et s.

Heintz G.F., Observations archéologiques à Achenheim-Bas de 1953 d̀ 1963, Cahiers Alsaciens d'Archéologie, d'Art et d'Histoire, 1964, p. $55-70$.

3 Die Altburg von Bundenbach (Allemagne, Rheinl. Pfalz)

Schindler R., Eine befestigte Höhensiedlung des 2/1 Jahrhunderts v. ch. im. Hunsrück. Die Altburg von Bundenbach, Trèves, 1977, 140 p., 97 fig., 9 pl.

4 Altenburg (Allemagne, Kr. Waldshut) Gersbach Eg., Altenburg (Waldschut) "Schwaben », Badische Fundberichte, 19, 1951, p. 168173. 
5 Alzey et ses environs (Allemagne, Rheinl. Pfalz) Benrens G., Germanisches Spätlatenegrab au Russelsheim am Main, Germania, 2, 1918, p. 4751.

Benhens G., Germanische Denkmaler der Frühzeit, I, Denkmaler des Wangionensgebietes, Francfort, 1923, 65 p., fig.

Stumpel B., Spätkeltische Funde im Westlichen Vorland von Alzey, Alzeyer Geschichtsblälter, Heft 9, 1972, p. 125-142.

6 Amplepuis, Le Terrail (Rhône)

Périchon R., Aperçu sur le site du Terrail, à Amplepuis (Rhône), Ogam, XIV, 1962, 1, p. 4784.

7 Argentat, Le Puy-du-Tour (Corrèze)

HatT J.-J., Quelques objets de La Tène III trouvés au Puy-du-Tour près d'Argentat (Corrèze), Bulletin de la Socièté Scientifique, Historique et Archéologique de la Corrèze, 63, 1941, p. 13-27.

Murat A. et J., 50 ans de recherches archéologiques à la station du Puy-du-Tour près d'Argentat (Corrèze), inventaire des découvertes de 1903 à 1953, Ogam, XIX, 1967, p. 369-396.

8 Aulnat (Puy-de-Dôme)

Périchon R., La céramique Gauloise avant la conquête, Dossiers de l'Archénlogie, 1974, 6, p. 9 18.

PÉrichon R., avec la collaboration de C. Ranchon et B. Sonial, Céramiques domestiques gauloises et gallo-romaines du Nord-Est du Massif central, Essai de typologie, Centre d'Études Foréziennes, Archéologie, $\mathrm{n}^{\circ}$ 6, Saint-Étienne, 1977, 280 p., 59 pl., 24 fig., 2 tableaux.

9 Bâle (Suisse)

Furger-Gunti A., Oppidum Basel-Munsterhügel, Jahrbuch der Schweizerische Gesellschaft für Ur- und Frühgeschichte, 58, 1974/1975, p. 78-111.

Furger-Gunti A., Der Murus Gallicus von Basel, Jahrbuch der schweizerische Gesellschaft für Ur- und Frühgeschichte, 63, 1980, p. 131-184.

MaJor E., Gallische Ansiedlung mit Gräberfeld bei Basel, 1940, Bâle, 211 p., 99 fig., 22 pl.

10 Bilina et Radovesice (Tchécoslovaquie, Bohême) Waldhauser J., Beitrag zum Studium der Keltischen Siedlungen Oppida und Gräberfelder in Böhmen, Colloque international, Les Mouve-

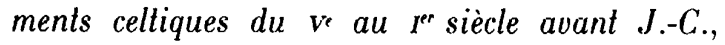
Nice, septembre 1976, p. 117-156.
11 Braubach (Allemagne, Rheinl. Pfalz)

Hans-Eckart J., Braubach und seine Umgebung in der Bronze und Eisenzeit, Bonner Jahrbücher, 177, 1977, p. 1-118.

12 Cavaillon (Vaucluse)

Dumoulin A., Puits et fosses de Cavaillon, Colline Saint-Jacques, Gallia, 23, 1965, p. 1-85.

13 Celles-les-Condé (Aisne)

Fagot P. et Chevallier R., Vestiges de La Tène II découverts par prospection aérienne à Celles-les-Condé (Aisne), les fouilles, la céramique domestique, Bulletin de la Socièté Préhistorique française, 55, 1958, p. 661-671.

14 Chalon-sur-Saône (Saône-et-Loire)

Armand-Calliat L., Dragage de la Saône aux abords de Châlon, Bulletin de la Société Nationale des Antiquaires de France, 1941, p. 215-219.

15 Chambles, Essalois (Loire)

Delporte H., La céramique d'Essalois, étude des séries conservées au musée de la Diana, Bulletin de la Diana, 35, 1957-1958, p. 180-190.

16 Clermont-Ferrand (Puy-de-Dôme)

Eychart P., L'oppidum des Côtes, ClermontFerrand (Puy-de-Dôme), Ogam, XIV, 1962, 1, p. $68-70$.

17 Ehl (Bas-Rhin)

Helmer L., La céramique de La Tène III à Ehl, Cahiers Alsaciens d'Archéologie, d'Art et d'Histoire, XIV, 1970, p. 11 et s.

18 Éprave, Le Trou de l'Ambre (Belgique, province de Namur)

Märien M. E., Le Trou de l'Ambre au Bois de Wérimont, Épraves, Musée Royaux d'Art et d'Histoire, Monographies d'Archéologie Nationale, $\mathrm{n}^{\circ}$ 4, Bruxelles, 1970, 273 p., 79 fig., 9 pl.

19 Eschen (Liechtenstein)

Eschen (Liechtenstein), Jahrbuch der Schweizerische Gesellschaft für Urgeschichte, 1943, p. 95 et pl. XXVIII.

20 Frohnhausen (Allemagne, Kr. Birkenfeld)

Haffner A., Mittellatenezeitliche Grabfunde aus Frohnhausen (Kr. Birkenfeld), Trierer Zeitschrift, 37, 1974, p. 49.

21 Genève (Suisse)

Paunier D., Céramique peinte de La Tène finale et matériel gallo-romain précoce trouvés sur l'oppidum de Genève, Genava, N.S., XXIII, 1975 , p. $55-125$. 
Paunier D., La céramique gallo-romaine de Genève de La Tène finale au royaume burgonde ( $\mathrm{I}^{\mathrm{er}} \mathrm{s}$. av. J.-C. $-\mathrm{v}^{\mathrm{e}} \mathrm{s}$. après J.-C.), Mémoires et documents de la Société d'Histoire et d'Archéologie de Genève, 9, Genève-Paris, 437 p., fig., pl.

22 Gergovie (Puy-de-Dôme)

Labrousse M., Les fouilles de Gergovie, campagnes de 1945 et 1946, Gallia, 6, 1948, p. 78-86.

23 Göblingen-Nospelt (Luxembourg)

Thill G., Die Keramik aus vier Spätlatenezeitlichen Brandgräbern von Göblingen-Nospelt, Hemecht, 2, 1967, p. 199-213.

24 Habsheim (Bas-Rhin)

Wolf J.-J., L'établissement de La Tène III de Habsheim Est, Bulletin du Musée Historique, 1970 , p. 7-15.

25 Hauviné (Ardennes)

Stas C., Un ensemble funéraire de La Tène III dans le site de l'abbaye de Vauclair (Aisne), Revue du Nord, 1971, III, p. 579-678, pl. IXVII.

26 Hesse supérieure, région de Starkenburg (Allemagne)

JoRns W., Farbige Spätlatenenzeit aus Starkenburg und Oberhessen, Fundchronik Land Hessen, p. 173-179.

27 Hüfingen (Allemagne, Bad. Württ., Kr. Donaueschingen)

Revellio P., Kastell Hüfingen, Germania, 10, 1926, p. 16-25.

Revellio P., Kastell Hüfingen, Germania, 11, 1927, p. 25-121.

28 Karlstein (Allemagne, Bavière)

Reinecke P., Grabfunde der dritten Latenestufe aus dem bayerischen Donautal, Altertümen unserer Heidnischen Vorzeit, V, Mainz, 1911, p. 288-291, pl. 51 et p. 364-369, pl. 63.

29 Ingelfingen (Allemagne, Bad-Württ., Kr. Kunselsau)

Kосн R., Siedlungsfunde der Latene und Kaiserzeit aus Ingelfingen (Kr. Kunselsau), Fundberichte aus Schwaben, NF, 19, 1971, p. 124-175.

30 La Lagaste (Aude)

Rancoule G., Ateliers de potiers et céramique indigène au I ${ }^{\text {er }}$ siècle avant J.-G., Revue Archéologique de Narbonnaise, III, 1970, p. 33-70.

31 Lijay (Loire)

Voir 8, Aulnat (Puy-de-Dôme).
32 Levroux (Indre)

Buchsenschutz O., Recherches sur l'habitat à l'âge du fer en Europe tempérée, Thèse de doctorat d'état, Université de Paris I, Panthéon-Sorbonne, Paris, 1982, dactylographiée.

33 Manching (Allemagne, Ldkr. Ingoldstadt) KaPPEL J., Die Graphitonkeramik von Manching, Die Ausgrabungen in Manching, 2, 1969, Wiesbaden.

MAIEr F., Bemalte Keramik von Manching, Die Ausgrabungen in Manching, 3, 1970, Wiesbaden.

Pingel V., Die Glatte Drehscheiben Keramik von Manching, Die Ausgrabungen in Manching, 4, 1971, Wiesbaden.

Sтöckli W. E., Die Grob- und Importkeramik von Manching, Die Ausgrabungen in Manching, 8, 1979, Wiesbaden.

34 Mont-Beuvray (Saône-et-Loire)

Cabotse J. et Périchon R., Les fouilles de Déchelette au Mont Beuvray en 1907, analyse du matériel recueilli, Ogam, XV, 1963, p. 161172.

35 Mont-Terrible (Suisse)

Quiquerez A., Monuments de l'Ancien Évêché de Bâle, Le Mont Terrible, avec notice historique sur les établissements des Romains dans le Jura Bernois, Société Jurassienne d'Émulation, Porrentruy, 1862 , pl. V.

36 Palatinat (Allemagne)

Voir note $\mathrm{n}^{0} 9$, p. 42.

37 Roanne (Loire)

Voir aussi 8, Aulnat (Puy-de-Dôme).

Caboste J. et Périchon R., Céramique de Roanne, Gallia, 24, 1966, p. 29-75.

38 Russelsheim-am-Main (Allemagne)

Voir 5, Alzey (Allemagne).

39 Sablonnière (Seine-et-Marne)

Voir 25, Hauviné (Ardennes).

40 Saint-Fréjoux (Corrèze)

Lintz G., Céramique de La Tène III à SaintFréjoux, Bulletin de la Société Scientifique Historique et Archéologique de la Corrèze, 94, 1972, p. $37-40$.

41 Saint-Marcel-de-Felines, Le Crêt Châtelard (Loire)

PÉrichon R., Premières observations sur la céramique peinte du Crêt Châtelard à SaintMarcel-de-Felines (Loire), Groupe de Recherches 
Archéologiques du département de la Loire, 1963, p. 23-31.

42 Saint-Maurice-aux-Forges (Meurthe-et-Moselle) Deyber A., Découverte d'un site gaulois de La Tène III au terroir de Saint-Maurice-aux-Forges (Meurthe-et-Moselle), Revue Archéologique de l'Est, XXIV, 1973, I, p. 129-143.

43 Saint-Maurice-sur-Loire, Jeuvre (Loire) Devauges J.-B., Les céramiques ocellées en Gaule de la fin de l'Indépendance à l'époque Gallo-romaine, Revue Archéologique de l'Est, XXXII, 1981, 1-2, p. 89-119.

44 Schänis (Suisse)

Schänis (Bez. Gaster, St-Gallen), Jahrbuch der Schweizerische Gesellschaft für Urgeschichte, 43, 1953 , p. 90-92.

45 Sissach-Brühl (Suisse)

Vogel V., Eine Nachgrabung im spätkeltichen Töpferbezirk Sissach-Brühl, Provincialia, Festschrift für Rudolf Laur Belart, Bâle, 1968, p. 619-631, 3 fig., 3 pl.

46 Stare-Hradisko (Tchécoslovaquie, Bohême) Meduna J., Das Keltische Oppidum StareHradisko in Mähren, Germania, 48, 1970, p. 3459.

47 Stradonice (Tchécoslovaquie, Bohême)

Pic J.-L., Le Hradischt de Stradonitz en Bohême, 1906, Leipzig.

48 Toulouse (Haute-Garonne)

Fouet G., Vases gaulois de la région Toulousaine, Gallia, 28, 1970, p. 11-33.

49 Tournus (Saône-et-Loire)

Perrin M. et Périchon R., Un site de La Tène III dans la Saône : Champsemard, près de Tournus (Saône-et-Loire) Gallia, 32, 1974, p. 225-242.

Perrin M., Essais de classification typologique préliminaire de la céramique de La Tène III découverte à Tournus (Saône-et-Loire), Découvertes Archéologiques en Tournugeois, 4, 1976, p. $27-68$.

50 Trèves (Allemagne, Rheinl. Pfalz)

HaffNer A., Zum Ende der Latenezeit im Mittelrheingebiet unter besonderer Berücksichtung des Trierer Landes, Archäologisches Korrespondenzblatt, 4, 1974, p. 59-72.

51 Verdun-sur-le-Doubs, Le Petit Chauvort (Saôneet-Loire)
Guillot A., La céramique peinte de La Tène du Petit Chauvort, Verdun-sur-le-Doubs (Saône-etLoire), La Physiophile, 74, 1971, p. 1-10.

52 Vernou-sur-Brenne (Indre-et-Loire)

Maugard R., Un fossé dépotoir à Vernou-surBrenne (Indre-et-Loire), II, étude du matériel céramique, Revue Archéologique du Centre, 16, 1977, p. 3-16.

53 Vienne (Isère)

Сhapotat G., Vienne Gauloise. Le matériel de La Tène III de la colline Sainte-Blandine à Vienne, 1-2, Lyon, Paris, de Boccard, 1970, 126 p., 34 fig., $56 \mathrm{pl}$.

54 Vindonissa (Suisse)

Ertlinger E. et Simonette Chr., Romische Keramik aus dem Schutthügel von Vindonissa, Veröffentlichungen des Gesellschaft pro Vindonissa, III, 1952, Birkhauser, Basel, p. 8-10.

55 Le Wetterau (Allemagne, Hesse)

Schönberger H., Die Spätlatenezeit in der Wetterau, Saalburg-Jahrbuch XI, 1952, p. 21$130,36 \mathrm{pl}$.

56 Yverdon (Suisse)

Srrrerding M., La céramique à l'époque de La Tène à Yverdon, fouille de 1961, Jahrbuch der Schweizerische Gesellschaft für Urgeschichte, 52, 1965, p. 100-111.

57 Sion (Meurthe-et-Moselle)

LEGENDRE J.-P., La céramique de l'époque Laténienne finale à Sion (Meurthe-et-Moselle), Mémoire de Maîtrise, Université de Nancy II, 1984, 97 p., 30 pl., no N84/8.

58 Villeneuve-Saint-Germain (Aisne)

Debord J., Premier bilan de huit années de fouilles à Villeneuve-Saint-Germain (Aisne) : 1973-1980, Vallée de l'Aisne, cinq années de fouilles protohistoriques, Revue archéologique de Picardie, numéro spécial, 1982, p. 213-264.

59 Condé-sur-Suippe et Variscourt (Aisne) Constantin C. et Ilett-Fleury B., Les installations de La Tène III de Condé-sur-SuippeVariscourt (Le vieux Reims), deux campagnes de fouilles 1976-1977, Vallée de l'Aisne, cinq années de fouilles protohistoriques, Revue Archéologique de Picardie, numéro spécial, 1982, p. 213-264.

60 Menil-Annelles et Ville-sur-Retourne (Ardennes)

Flouest J.-L., L'évolution des formes de la 
céramique dans les cimetières de Menil-Annelles et Ville-sur-Retourne de La Tène II au galloromain précoce, Revue du Nord, numéro spécial hors série, Les Celtes en Belgique et dans le Nord de la France, les fortifications de l'Age du Fer; Acte du sixième colloque tenu à Bavay et Mons, éd. par A. Cahen-Delhaye, A. Duval, G. LemanDelerive, P. Leman, 1984, p. 51-57.

\section{Pont-sur-Yonne (Yonne)}

Prampart J.-Y., Le monument funéraire du bas des Renardières à Pont-sur-Yonne (Yonne), époque de La Tène, L'Age du Fer en France septentrionale, Mémoires de la Société Archéologique Champenoise - 2, supplément au Bulletin $\mathrm{n}^{\circ}$ 1, 1981, p. 263-289.

62 Vaires-sur-Marne (Seine-et-Marne)

Brulard A. et Drounot C., Le site de La Tène finale de l'Ile-Ronde à Vaires-sur-Marne (Seineet-Marne), l'Age du Fer en France septentrionale, Mémoires de la Société Archéologique Champenoise - 2, supplément au Bulletin $\mathrm{n}^{\circ} 1,1981$, p. 346364.

M. D. et A. D. 